\title{
MicroRNA-138-5p Regulates Hippocampal Neuroinflammation and Cognitive Impairment by NLRP3/Caspase-I Signaling Pathway in Rats
}

This article was published in the following Dove Press journal: Journal of Inflammation Research

\author{
Xiaojin Feng $\mathbb{D}^{1,2}$ \\ Jialing $\mathrm{Hu}^{\prime}$ \\ Fenfang Zhan' \\ Deqiang Luo $\mathbb{D}^{3}$ \\ Fuzhou Hua ${ }^{1,2}$ \\ Guohai $X^{1,2}$
}

'Department of Anesthesiology, The Second Affiliated Hospital of Nanchang University, Nanchang, Jiangxi, 330006, People's Republic of China; ${ }^{2}$ Key Laboratory of Anesthesiology of Jiangx Province, Nanchang, Jiangxi 330006, People's Republic of China; ${ }^{3}$ Department of Intensive Care Unit, The First Affiliated Hospital of Nanchang University, Nanchang, Jiangxi, 330006, People's Republic of China
Correspondence: Guohai Xu; Fuzhou

Hua

Department of Anesthesiology, The Second Affiliated Hospital of Nanchang University, Nanchang, Jiangxi, 330006,

People's Republic of China

Tel +86-79l-86299|3|

Email xuguohail@sina.com;

4I5I34607@qq.com
Purpose: Neuroinflammation is an essential causative factor in the pathogenesis and progression of cognitive impairment. The present study aims to evaluate the critical role of microRNA-138-5p (miR-138-5p) in hippocampal neuroinflammation and cognitive impairment through the NLRP3/caspase-1 signaling pathway in rats.

Material and Methods: We established the cognitive impairment rat model and RM (Rat microglia) microglial cellular inflammation model by intracerebroventricular (icv) injection or stimulation of lipopolysaccharide (LPS). Morris water maze (MWM) and Y-maze tests were performed to assess the cognitive behaviors. Quantitative real-time polymerase chain reaction (qRT-PCR), Enzyme-linked immune-sorbent assay (ELISA) and Western blot analysis were utilized to evaluate mRNA or protein expression. Bioinformatic analysis and dualluciferase reporter gene assay were performed to verify the targeting relationship between NLRP3 and miR-138-5p. Besides, Hematoxylin and eosin (H\&E) staining and immunohistochemistry were applied to observe the neuronal morphology and detect the positive cells of the hippocampus, respectively.

Results: Compared to the control groups, LPS-treated rats exhibited significantly impaired learning and memory in MWM and Y-maze tests. The expression of NLRP3, caspase-1 and pro-inflammation cytokines (IL-1 $\beta$ and IL-18) were upregulated, while miR-138-5p was downregulated both in rat hippocampus and RM cells treated with LPS. MiR-138-5p is downregulated in microarray data of cognitive impairment animals and could directly target the 3'-UTR of NLRP3. Furthermore, upregulation of miR-138-5p improved impaired cognitive functions, while inhibited hippocampal neuroinflammation demonstrated by decreased expression of NLRP3/caspase-1 axis, pro-inflammation cytokines and microglial activation. This study demonstrates for the first time that miR-138-5p suppresses the hippocampal NLRP3/caspase-1 signaling pathway activation in cognition impaired rats.

Conclusion: The low expression of miR-138-5p after LPS administration may contribute to the activation of the NLRP3/caspase-1 pathway, leading to hippocampal neuroinflammation and cognitive impairment in rat models. These findings indicate a promising therapeutic avenue for cognitive disorders.

Keywords: microRNA-138-5p, NLRP3/caspase-1, neuroinflammation, cognitive impairment, microglial activation

\section{Introduction}

Cognitive impairment is one of the major symptoms in most neurodegenerative disorders, including Parkinson's disease (PD), Alzheimer's disease (AD) and Huntington's disease (HD), as well as perioperative neurocognitive disorders 
(PND). ${ }^{1}$ It is characterized by a decline of cognitive functions, such as memory, attention and information processing, which is directly related to the morbidity and disability of the diseases, bringing a considerable socioeconomic burden to countries worldwide. $^{2}$ Neuroinflammation has been reported to participate in cognitive impairment associated with some neurodegenerative diseases, and clinical as well as experimental evidence has shown that anti-inflammation treatment could alleviate cognitive impairment. ${ }^{3-5}$ The common characteristic of these diseases is progressive neuronal loss and impaired neuronal function, lacking effective treatments. ${ }^{6}$ Lipopolysaccharide (LPS), a major component of various gram-negative bacteria, has been shown to induce hippocampal neuroinflammation and cognitive decline, is generally utilized to activate inflammation and prepared cognitive impairment model in vitro or in vivo. ${ }^{7-10}$ Although many studies have indicated that diverse pathological processes are associated with deleterious neuroinflammation of cognitive disorders, the exact mechanism underlying these diseases is still elusive.

Microglia are the prominent innate immune cells of the central nervous system (CNS), which play a pivotal role in physiological and pathological conditions. ${ }^{11,12}$ They mediate immune responses by pattern recognition receptors, including Toll-like receptors and NOD-like receptors (NLRs). ${ }^{13}$ Overactivated microglia cause neuronal loss and injury via neurotoxicity, resulting in declined synaptic function. ${ }^{11}$ NLR family pyrin domain-containing-3 (NLRP3) is a typical representative of the NLRs family, which plays an essential role in triggering inflammasome-mediated neuroinflammation and the pathogenesis of cognitive impairment. ${ }^{14}$ NLRP3 inflammasome consists of NLRP3, apoptosis-associated speck-like protein (ASC) and pro-caspase-1. This inflammasome modulates the activation of caspase- 1 that controls the secretion of the pro-inflammatory cytokines, such as IL-1 $\beta$ and IL-18. ${ }^{15}$ Importantly, NLRP3/caspase-1 signaling pathway has a fundamental biological role in innate immune defense against microbial pathogens. ${ }^{16}$ Clinical evidence revealed that the blood expression levels of the abovementioned protein (NLRP3, pro-inflammatory cytokines) were promoted in AD patients. ${ }^{17,18}$ Moreover, recent studies have indicated that NLRP3 was upregulated in the hippocampus of cognitive impairment models, and the impaired function can be reversed by the inhibition of NLRP3 expression. ${ }^{19-21}$ Thus, the NLRP3 inflammasome may be a viable target to interrupt the pathogenesis of cognitive impairment, although there is no exact activation mechanism.

MicroRNAs (miRNAs) are a family of non-coding RNAs with 19-22 nucleotides length, which regulate target gene expression by messenger RNAs (mRNAs) degradation or translational repression. Emerging evidence demonstrates that aberrant expression of miRNAs, such as proinflammatory (miR-196a, miR-34b and miR-126), antiinflammatory (miR-9, miR-146a and miR-22) and mixed immunomodulatory (let-7 family) miRNAs, are critical in inflammatory and CNS diseases. ${ }^{22}$ MiR-138-5p, as a member of miRNAs, is closely associated with various human diseases, including several types of cancers, ${ }^{23-25}$ obesity, $^{26}$ and especially for inflammatory diseases. ${ }^{27,28}$ of inflammatory illnesses, miR-138-5p has been found downregulated in acute myocardial infarction model induced by hypoxia/reoxygenation ${ }^{27}$ and chondrocytes of osteoarthritis model caused by IL-1 $\beta .^{28}$ Conversely, miR-138-5p was overexpressed in pulmonary arterial smooth muscle cells/ lungs in patients or rats of pulmonary arterial hypertension, which could be alleviated by inhibiting the miR-138-5p expression. $^{29}$ However, little is known about the role of miR-138-5p in hippocampus-related cognitive impairment induced by LPS. Furthermore, our bioinformatics analysis results showed that miR-138-5p expression was downregulated in the hippocampus of the PND model. Then we found that there is a binding site between NLRP3 and miR-138-5p by miRDB online prediction software. Therefore, given the crucial role of miR-138-5p on the inflammatory response, it is attractive to clarify the underlying mechanism of miR$138-5 p$ in neuroinflammation mediated-cognitive impairment, and the role of miR-138-5p in modulating the NLRP3/caspase-1 pathway activation.

To our knowledge, this is the first study that identified the crucial role of miR-138-5p in cognitive impairment induced by LPS. The present study identified that miR$138-5$ p could attenuate early cognitive impairment by regulating the NLRP3/caspase-1 signaling pathway in LPSinduced hippocampal neuroinflammation. Our study may contribute to a better understanding of the molecular mechanisms underlying NLRP3 activation and provide a novel target for cognitive impairment therapy.

\section{Materials and Methods}

\section{Animals and Ethic Statement}

Adult male Sprague-Dawley (SD) rats (200-300 g) were obtained from the Animal Center of Nanchang University. 
They were raised in plastic cages under a 12-h light-dark cycle with food and water ad libitum. The room temperature (RT) was controlled at $24^{\circ} \mathrm{C} \pm 1{ }^{\circ} \mathrm{C}$, and relative humidity was maintained at $50 \%-60 \%$. We attempted to minimize the number of rats and pain. All experimental procedures were approved by the Institutional Animal Care and Use Committee of Nanchang University (NO. 2016020) and complied with the International Association for the Study of Pain Guidelines.

\section{Intracerebroventricular Injection}

Intracerebroventricular (icv) injection of drugs was performed as previously described. ${ }^{30,31}$ Briefly, rats were anesthetized with sodium pentobarbitone by intraperitoneal (i.p.) injection $(45 \mathrm{mg} / \mathrm{kg})$ and then fixed in a stereotactic frame (RWD Life Science, Shenzhen, China). Subsequently, the skin of the heads was incised along the midline for about $1 \mathrm{~cm}$. Referring to brain atlas, the left lateral ventricle was used as the administration target (millimeters from bregma): anterior-posterior = $1.0 \mathrm{~mm}$, mediolateral $=1.5 \mathrm{~mm}$, and dorsoventral $=$ $-3.8 \mathrm{~mm}$. A microinjector was used to infuse the compounds $(10 \mu \mathrm{L})$ with a rate of $1 \mu \mathrm{L} / \mathrm{min}$, and the syringe was held in place for $10 \mathrm{~min}$ after infusion. The penicillin powder was used for local disinfection. Then the skin was sutured, and the rats were returned to the breeding room for recovery.

\section{Experimental Design and Establishment of Model}

The first experiment was designed to examine the effect of inflammation on cognitive behaviors and NLRP3 activation in the rat hippocampus. Thirty-four rats were randomly assigned into two groups: the control group and the LPS group, which received saline $(10 \mu \mathrm{L})$ and LPS $(50 \mu \mathrm{g}$ in $10 \mu \mathrm{L}$ saline), ${ }^{32}$ respectively. LPS (Escherichia coli, serotype O55: B5) was purchased from Sigma-Aldrich (St. Louis, MO, USA). Some rats of each group were trained for behavioral tests 1 day before and 1, 3 and 7 days after LPS/saline injection ( $\mathrm{n}=5$ per group). The timeline was shown in Figure 1A. The remaining rats were sacrificed after $6 \mathrm{~h}, 12 \mathrm{~h}$ and $24 \mathrm{~h}$ after injection (n $=6$ per group/time points), and the hippocampus was collected for quantitative real-time polymerase chain reaction (qRT-PCR), Enzyme-linked immune-sorbent assay (ELISA) and Western blot analysis $(\mathrm{n}=6$ per group).
A subsequent experiment was performed to assess the effect of miR-138-5p on cognitive behaviors and hippocampal neuroinflammation in rats. Sixty-six rats were randomly assigned into six experimental groups $(\mathrm{n}=$ 11 per group): control group, LPS group, LPS + antagomir group, LPS + antagomir negative control (NC) group, LPS + agomir group, and LPS + agomir NC group. Rats in the control and LPS group received saline and LPS $(50 \mu \mathrm{g})$, respectively. According to the manufacturers' instructions (RiboBio Co., Ltd., Guangzhou, China) and our pre-experiment results (Data was not shown), rats in the LPS + antagomir/agomir group received miR-138-5p antagomir/agomir (2.5 $\mathrm{nM}$ in 10 $\mu \mathrm{L}$ ) in the hippocampus $72 \mathrm{~h}$ before LPS injection, while rats in the LPS + antagomir/agomir NC group received respective $\mathrm{NC}$ reagents. Like the previous protocol, thirty rats were performed behavioral tests on 1 day before and 1, 3 and 7 days after LPS injection ( $n=5$ per group). The timeline was shown in Figure 6A. Rats were sacrificed by exsanguination under anesthesia after the last behavioral test, and hippocampal tissues were collected for Hematoxylin and eosin (H\&E) staining and immunohistochemistry. The remaining thirty-six rats were sacrificed at $24 \mathrm{~h}$ after LPS injection. The hippocampi were collected for qRT-PCR, ELISA and Western blot analysis ( $n=6$ per group).

\section{Behavioral Tests}

Morris water-maze (MWM) test was performed to evaluate the long-term spatial learning and memory as described by Morris with slight modifications. ${ }^{33,34}$ The MWM apparatus (XR-XM101, Shanghai Xinruan, China) consists of a circular pool with a diameter of $150 \mathrm{~cm}$ and a height of $60 \mathrm{~cm}$, which is split into four quadrants of an equal area filled with tap water $\left(30 \mathrm{~cm}\right.$ in-depth, $\left.25^{\circ} \mathrm{C}\right)$. A circular platform (12 $\mathrm{cm}$ in diameter) is $3 \mathrm{~cm}$ below the water surface in the center of one quadrant. The MWW task includes a five consecutive daily acquisition trial and a probe trial. During the acquisition trial test, the rat was released into the water facing the wall and allowed to explore the hidden platform for $60 \mathrm{~s}$ and remain on it for $10 \mathrm{~s}$. If the rat cannot approach the platform within $60 \mathrm{~s}$, it was guided to the platform for an additional $10 \mathrm{~s}$. The process was repeated for all four quadrants, and the escape latency was recorded. At the end of the acquisition trial test, the platform was removed, and a probe trial was conducted. The number of crossings the platform within $60 \mathrm{~s}$ and the time spent in the target quadrant was also 

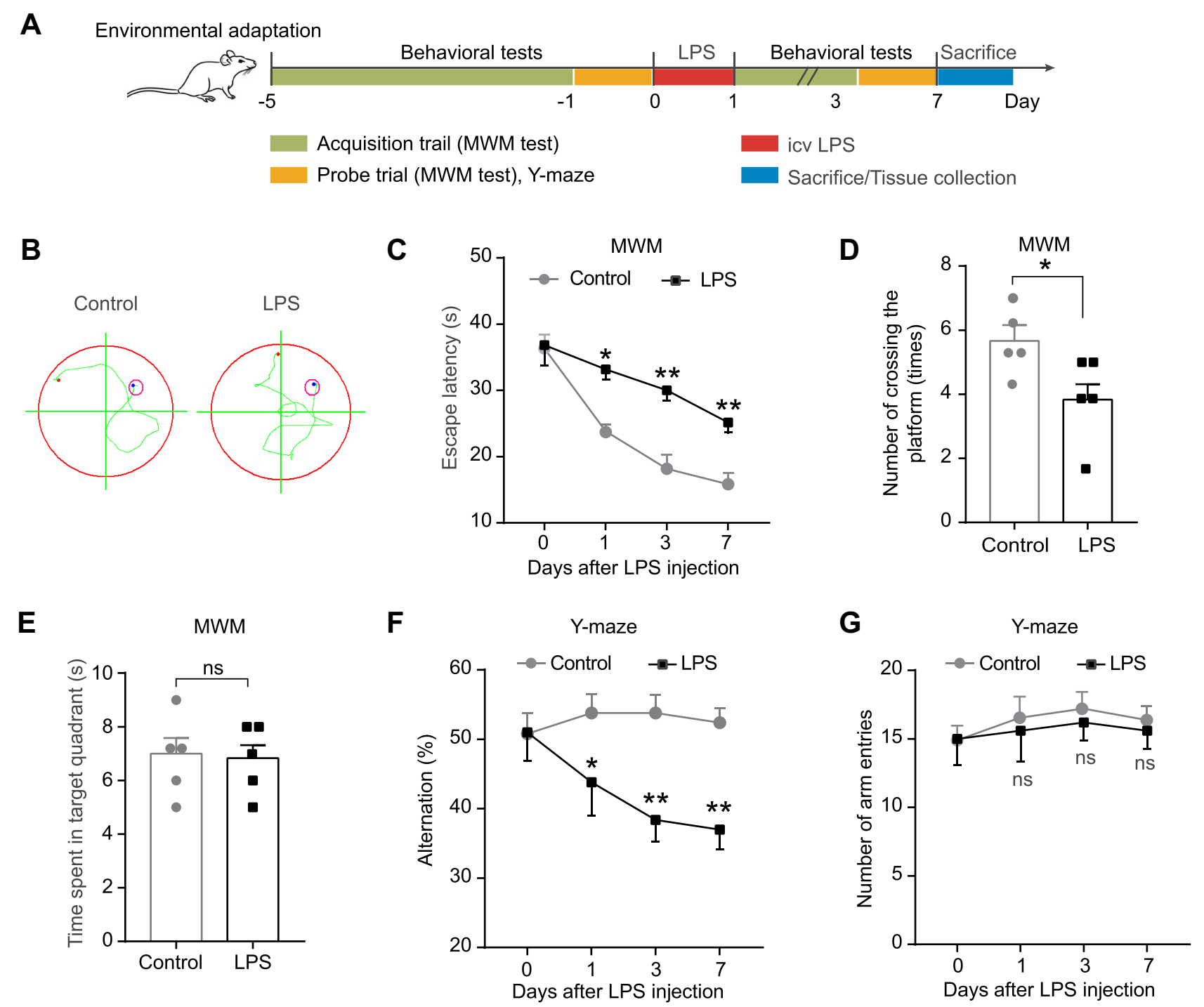

Figure I Effect of LPS on spatial learning and memory ability of rats in MWM test and Y-maze test. (A) Experimental protocol and timeline. (B) Representative swimming path on day 7 after LPS injection in MWM test. Effect of LPS on (C) escape latency, (D) number of crossing the platform, and (E) time spent in the target quadrant in MWM test. Effect of LPS on $(\mathbf{F})$ alternation and $(\mathbf{G})$ number of arm entries in Y-maze test. Data were presented as mean \pm SEM. $n=5$ per group. ${ }^{*} p<0.05$, $* * p<0.0$.

Abbreviations: icv, intracerebroventricular; LPS, lipopolysaccharide; MWM, Morris water-maze; ns, not significant.

analyzed. The MWM test was carried out from 8:00 to 12:00 am.

Y-maze test was used to examine the short-term spatial learning and memory as described. ${ }^{35}$ The Y-maze apparatus containing three equal-length black arms (A, B and C) with a $120^{\circ}$ angle between each other. The length, width and height of each arm were $50 \mathrm{~cm}, 16 \mathrm{~cm}$ and $32 \mathrm{~cm}$, respectively. At the beginning of the test, the rat was accustomed to the device for $30 \mathrm{~min}$. It was put individually on the center of the Y-maze and allowed to run freely for $5 \mathrm{~min}$, then the number of alternations and the number of arm entries were recorded. Alternations were calculated when a rat entered consecutive arms, such as ABC, BCA, but not CBC. The alternation is calculated by the following formula: number of alternations/(total number of arm entries - 2)\%. At the end of each test, the maze was treated with $75 \%$ alcohol before the next test. The Y-maze test was conducted between 14:00 and 17:00. All behavioral tests were performed in a soundproof with a blinded manner.

\section{Tissue Collection}

For qRT-PCR, ELISA and Western blot analysis, rats were deeply anesthetized with sodium pentobarbitone (i.p., $45 \mathrm{mg} / \mathrm{kg}$ ) and transcardially perfused with $0.1 \mathrm{M}$ icecold phosphate-buffered saline (PBS, $\mathrm{PH}=7.3,150 \mathrm{~mL}$ ). 
The brain was subsequently dissected by craniotomy. Immediately, the hippocampus tissues were dissociated and put into cryogenic tubes, then were quickly removed to liquid nitrogen and then transferred to $-80^{\circ} \mathrm{C}$ until use. For H\&E staining and immunohistochemistry, the rats were perfused with PBS $(150 \mathrm{~mL})$ followed by $4 \%$ paraformaldehyde $(\mathrm{PH}=7.3,200 \mathrm{~mL})$. The hippocampal tissues were fixed in $4 \%$ paraformaldehyde for $24 \mathrm{~h}$.

\section{Cell Culture and Grouping}

The immortalized RM (Rat microglia) microglial cell line was purchased from the Tongpai Biotechnology (Shanghai, China). It was cultured in Dulbecco's modified Eagle's medium supplemented with $10 \%$ fetal bovine serum (Gibco, Carlsbad, USA) at $37^{\circ} \mathrm{C}$ under $95 \%$ air and $5 \% \mathrm{CO}_{2}$. In the preliminary experiment, the cells were randomly assigned into the control and the LPS (1 $\mu \mathrm{g} / \mathrm{mL}$ ) groups. Given previous studies demonstrate that a concentration of $1 \mu \mathrm{g} / \mathrm{mL}$ LPS induces a significant inflammatory response in both immortalized and primary microglia, ${ }^{36}$ we stimulated RM microglia cells with $1 \mu \mathrm{g} /$ mL LPS in this study.

In the subsequent cell transfection experiment, the cells were randomly assigned into six groups: control, LPS, LPS + miR-138-5p inhibitor (LPS + inhibitor), LPS + miR-138-5p inhibitor NC (LPS + inhibitor NC), LPS + miR-138-5p mimic (LPS + mimic) and LPS + miR-138$5 p$ mimic NC (LPS + mimic NC). RM cells were seeded in 6-well plates and reached $30-50 \%$ confluency before the transfection. According to manufacturers' instructions (RiboBio Co., Ltd., Guangzhou, China), $100 \mathrm{nM}$ working concentration of miR-138-5p inhibitor, miR138-5p mimic, miR-138-5p inhibitor NC or miR-138-5p mimic $\mathrm{NC}$ was mixed with its riboFECT $\mathrm{T}^{\mathrm{tm}} \mathrm{CP}$ Reagent, respectively; and was added to the 6-well plates. After 72 h post-transfection, the RM cells were stimulated by LPS $(1 \mu \mathrm{g} / \mathrm{mL}$ ) for $6 \mathrm{~h}$ and collected for RNA or protein isolation. The supernatant medium was collected for ELISA.

\section{qRT-PCR}

Total RNA was isolated from hippocampal tissues and RM microglial cells using TRIZol (Invitrogen, CA, USA). Total RNA extraction and reverse transcription to cDNA (1 $\mu \mathrm{g}$ RNA) were carried out with a Primescript ${ }^{\mathrm{TM}}$ RT reagent kit (RR037A, Takara, Japan) on an ABI Veriti PCR (Applied Biosystems, USA). The reaction of qRT-PCR for each gene was detected in triplicate using the SYBR Premix Ex Taq ${ }^{\text {TM }}$ II kit (RR820A, Takara, Japan) on an ABI 7900 thermocycler (Applied Biosystems, USA) according to the manufacturer's instructions. The specific primer sequences of mRNAs are listed in Table 1. The specific primers of U6 and miR-138-5p (bulge-loop RT and qRT-PCR primers) were designed by RiboBio (RiboBio Co., Ltd, Guangzhou, China). $\beta$-actin and U6 were used as the internal control for mRNA and miR-138-5p, respectively. Reaction without any template was served as negative controls. Relative mRNA expression was calculated by the $2^{-\Delta \Delta \mathrm{Ct}}$ method. ${ }^{37}$

Table I Specific Primers Sequences of mRNAs

\begin{tabular}{|l|l|l|l|}
\hline Gene & $\begin{array}{l}\text { GenBank } \\
\text { Accession }\end{array}$ & Primer Sequences (5'-3') & Product Size (bp) \\
\hline NLRP3 & NM_00II91642.I & $\begin{array}{l}\text { F: CCAGGAGTTCTTTGCGGCTA } \\
\text { R: GCCTTTTTCGAACTTGCCGT }\end{array}$ & 145 \\
\hline Caspase-I & NM_012762.3 & $\begin{array}{l}\text { F: CTGGAGCTTCAGTCAGGTCC } \\
\text { R: CTTGAGGGAACCACTCGGTC }\end{array}$ & 141 \\
\hline IL-I $\beta$ & NM_03I5I2.2 & $\begin{array}{l}\text { F: CTTGACTTGGGCTGTCCAGA } \\
\text { R: ACGGGCAAGACATAGGTAGC }\end{array}$ & 133 \\
\hline IL-I8 & NM_019165.2 & $\begin{array}{l}\text { F: CAACCGCAGTAATACGGAGC } \\
\text { R: GATTCGTTGGCTGTTCGGTC }\end{array}$ & 106 \\
\hline Ibal & NM_017I96.3 & $\begin{array}{l}\text { F: CAACAAGCACTTCCTCGATGATC } \\
\text { R: TGAAGGCCTCCAGTTTGGACT }\end{array}$ & 165 \\
\hline -actin & NM_03II44.3 & $\begin{array}{l}\text { F: TGTCACCAACTGGGACGATA } \\
\text { R: GGGGTGTTGAAGGTCTCAAA }\end{array}$ & \\
\hline
\end{tabular}

Abbreviations: F, forward; R, reverse; bp, base pair. 


\section{ELISA}

The concentrations of pro-inflammation cytokines (IL-1 $\beta$ and IL-18) in the rat hippocampal tissues and RM microglial cells were detected by ELISA assays using ELISA kits (CUSABIO, Wuhan, China) according to the manufacture's protocols.

\section{Western Blot Analysis}

Hippocampal tissues and RM microglial cells were homogenized in RIPA lysis buffer containing PMSF for $1 \mathrm{~h}$ on ice. The supernatants were collected after centrifuged at $4^{\circ} \mathrm{C}$ for 15 min with 12,000 relative centrifugal force (rcf). A bicinchoninic acid protein assay kit (TIANGEN, Beijing, China) was used to measure the protein concentration. Then, the protein samples $(30-50 \mu \mathrm{g})$ were resolved using SDS-polyacrylamide gel electrophoresis $(8-12 \%)$ and transferred to PVDF membranes $(0.22 \mu \mathrm{M})$. After blocking with 5\% non-fat skimmed milk for $2 \mathrm{~h}$ at RT, the membranes were incubated with the following primary antibodies overnight at $4{ }^{\circ} \mathrm{C}$ : rabbit polyclonal anti-NLRP3 (1:500, NBP2-12446, NOVUS, USA), rabbit polyclonal anti-caspase-1 (1:1000, ab1872, Abcam, USA), rabbit polyclonal anti-GAPDH (1:5000, 10494-1-AP, proteintech, China) and rabbit polyclonal anti-Iba (1:1000, WL02406, Wanleibio, China). Subsequently, membranes were incubated with horseradish peroxidase-conjugated secondary antibody (1:5000, proteintech, China) for $2 \mathrm{~h}$ at RT. Bands were visualized with a Bio-Rad Gel Doc EZ imager (Bio-Rad, USA), and the intensity was analyzed by Image J software (NIH Image analysis website: http://rsb.info.nih.gov/ij/). GAPDH was used as an internal reference.

\section{Bioinformatics Analysis and Dual-Luciferase Reporter Gene Assay}

We utilized miRDB online prediction software (http:// mirdb.org/index.html) to screen the potential miRNAs that could bind to 3 '-UTR sequence of NLRP3, and predicted the binding site between NLRP3 and miR-138-5p. The basic information and species conservativeness of miR$138-5 \mathrm{p}$ were obtained from the UCSC Genome Bioinformatics website (http://genome.ucsc.edu/). The miRNAs expression microarray data (GSE95070) of the cognitive impairment animal model was downloaded from the National Center for Biotechnology Information (NCBI) Gene Expression Omnibus (GEO) database (https://www. ncbi.nlm.nih.gov/geo/) of platform GLP19117. The identification of differentially expressed miRNAs was determined by the gplots and heatmap packages in the $\mathrm{R}$ platform. P-value $<0.05$ were considered as differentially expressed genes.

We performed a luciferase activity assay using a Dual-Luciferase Reporter Assay Kit (Promega, Wisconsin, USA) according to the manufacturer's instructions. Briefly, the wild-type (WT) or mutant (MUT) of NLRP3 3'-UTR luciferase reporter constructs were co-transfected into $293 \mathrm{~T}$ cells with miR-138-5p mimic or miR-138-5p mimic NC using Lipofectamine 3000. After $48 \mathrm{~h}$ of transfection, the cells were collected and examined with a dual-luciferase reporter assay system (Promega, Wisconsin, USA).

\section{Cell Apoptosis Analysis}

Cell apoptosis analysis was performed using a Hoechst 33342/PI (Propidium iodide) double stain kit (Solarbio Life Science, Wuhan, China). The cell culture medium for RM cells was removed after different treatments, and then Hoechst and PI staining solutions were added. After incubation for $20 \mathrm{~min}$ in the dark at $4^{\circ} \mathrm{C}$, cells are washed for once with PBS. Cell apoptosis was observed by a fluorescent microscope (Olympus, Tokyo, Japan) and analyzed by Image $\mathrm{J}$ software.

\section{H\&E Staining and Immunohistochemistry}

H\&E staining was used to observe the neuronal morphology and damage, while immunohistochemistry was performed to detect the activation of NLRP3 and microglia in the rat hippocampus. The brain tissues were embedded in paraffin, and $5-\mu \mathrm{m}$ thick brain sections were prepared using a slicer (RM2235, Leica, Germany). After dewaxed and rehydrated by xylene and ethanol, the slides were stained with H\&E staining or immunohistochemistry. $\mathrm{H} \& \mathrm{E}$ staining was conducted on $5 \mu \mathrm{m}$ sections using standard H\&E staining protocol. For immunohistochemistry, the sections were subjected to induced antigen retrieval in citrate buffer at $100^{\circ} \mathrm{C}(0.01 \mathrm{M}, \mathrm{pH}=6.0)$, and then immersed in 3\% hydrogen peroxide solution for $10 \mathrm{~min}$ at RT to quench endogenous peroxidase activity. After blocking with $5 \%$ bull serum albumin, the tissues were incubated with anti-NLRP3 antibody (1:50, BA3677, Boster, Wuhan, China) or anti-Iba-1 antibody (1:500, ab178846, Abcam, USA) overnight at $4^{\circ} \mathrm{C}$. Afterward, the sections were rinsed with PBS three times and incubated with antirabbit IgG secondary antibodies (1:200, Boster, Wuhan, China) at RT for $1 \mathrm{~h}$, and then were viewed by 
3,3'-diaminobenzidine solution and counterstained with Hematoxylin. The sections were visualized and analyzed by a light microscope (Olympus, Tokyo, Japan) equipped with an imaging system and Image J software.

\section{Statistical Analysis}

Data are represented as mean \pm standard error mean (SEM). The statistical analyses were carried out by using GraphPad Prism 7 (GraphPad Software, Inc., La Jolla, USA) and SPSS 22.0 (SPSS Inc., Chicago, IL, USA). Shapiro-Wilk test and Levene's test were used to evaluate the normal distribution and the homogeneity of variance of data, respectively. Student's $t$ test or Mann-Whitney U was used for the comparison between two groups. Comparison of the escaping latency, alternation and number of entries were analyzed by repeated-measures one-way analysis of variance followed by post hoc of Bonferroni test or Dunnett's test. $p<0.05$ was considered statistically significant.

\section{Results}

\section{LPS Induces Cognitive Impairment of Rats in MWM Test and Y-Maze Test}

To clarify the effect of LPS injection on the learning and memory of rats, the MWM test and Y-maze test were performed on 1 day before and 1, 3, and 7 days after LPS/saline injection. No rats in each group died during the injection or the intended observation period after the injection. The experimental outline was summarized in Figure 1A. The MWM test result showed that compared with the control group, LPS significantly prolong the escape latency on day 1, 3 and 7 ( $p<0.05-0.01$, Figure 1B-C), and decreased the number of crossing the platform of rats on day 7 ( $p<0.05$, Figure 1D). However, there was no significant difference in the time spent in the target quadrant between the control and LPS-treated rats $(p>$ 0.05 , Figure $1 \mathrm{E})$. To further extend this result, we assessed the immediate spatial working memory of LPS-treated rats by the Y-maze test. As shown in Figure 1F-G, LPS significantly decreased the alternation in rats compared with the control group on days 1,3 and $7(p<0.05-0.01)$, while leading to no difference in the number of arm entries between the two groups $(p>0.05)$. These results show that LPS could impair the memory and learning of rats, suggesting a successful establishment of an animal model of cognitive impairment.

\section{LPS Increases the Expression of NLRP3/ Caspase-I and Pro-Inflammatory Cytokines in the Hippocampus of Rats}

Neuroinflammation plays an essential role in regulating memory impairment. A previous study revealed that the level of several pro-inflammatory cytokines (IL-1 $\beta$, IL-6 and TNF- $\alpha$ ) rises progressively within 6 hours in the hippocampus after LPS injection, ${ }^{7}$ thus, we harvested hippocampal tissues at $6 \mathrm{~h}, 12 \mathrm{~h}$ and $24 \mathrm{~h}$ after LPS treatment in this study. The qRT-PCR result indicated that compared with the control group, the mRNA expression of NLRP3, caspase-1, pro-inflammatory cytokines (IL-1 $\beta$ and IL-18) were upregulated in LPS-treated rats at $6 \mathrm{~h}, 12 \mathrm{~h}$ and $24 \mathrm{~h}(p<0.01-0.001$, Figure $2 \mathrm{~A})$. Meanwhile, the ELISA result showed that the release of IL-1 $\beta$ and IL-18 were significantly increased in LPStreated rats compared with the control rats $(p<0.001$, Figure 2B). Furthermore, as shown in Figure $2 \mathrm{C}$-E, the protein expression of NLRP3 and caspase-1 were enhanced by LPS $(p<0.01-0.001)$. There was no significance between different time points $(6 \mathrm{~h}, 12 \mathrm{~h}$ and 24 h) $(p>0.05)$. These results suggest that the activation of NLRP3/caspase-1 signaling pathway contributes to LPSinduced hippocampal neuroinflammation in rats.

\section{Low Expression of MiR-138-5p Was Found in Hippocampus of LPS-Treated Rats}

Increasing evidence indicates that post-transcriptional regulation by miRNAs is vital in the pathogenesis of neurodegenerative diseases and cognitive dysfunction. ${ }^{38}$ Next, we focus our attention on the abnormal NLRP3 inflammasome to explore the mechanism underlying neuroinflammation of cognitive dysfunction. As shown in Figure 3A, the bioinformatics online website miRDB predicted three miRNAs that could bind to NLRP3 in rats, of which the ranking order is miR-138-5p (79), miR-325-5p (57) and miR-370-3p (50) based on the target score. MiR-138-5p, the top-ranking miRNA among these miRNAs, is highly conserved across species based on the basic information of chromosome location, base sequence and species conservativeness (Figure 3B). Furthermore, we obtained a dataset of miRNA profiling by the array (GSE95070) containing five pairs of hippocampal tissues from the cognitive impairment model and control animals, which the 


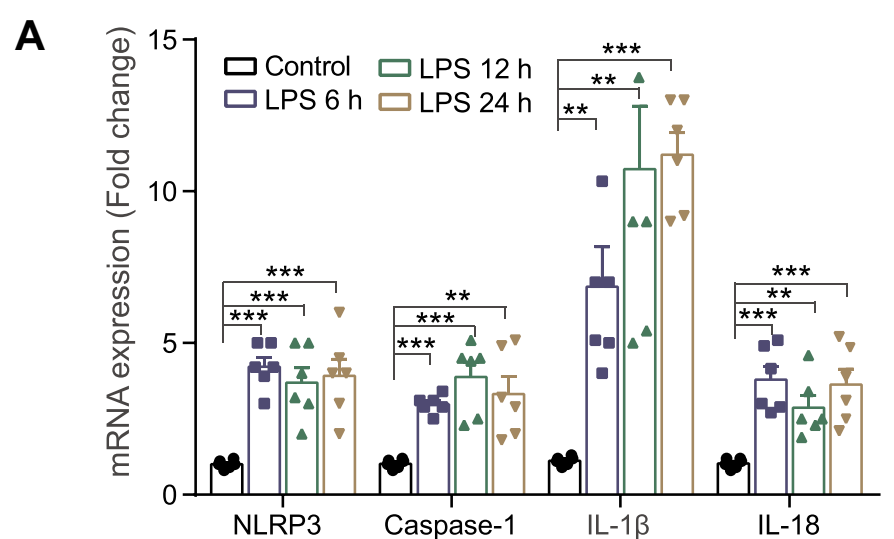

C

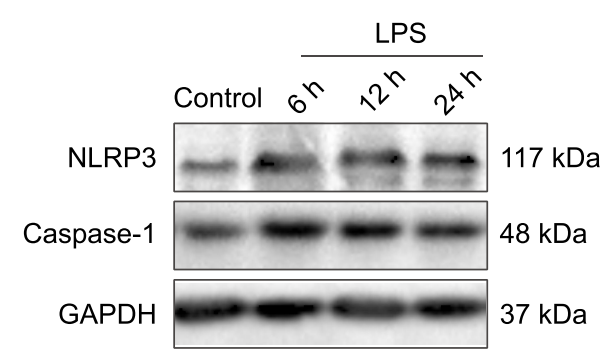

B

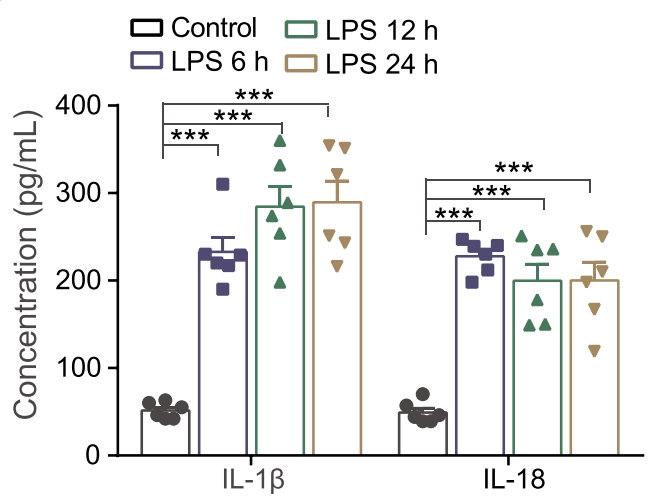

E

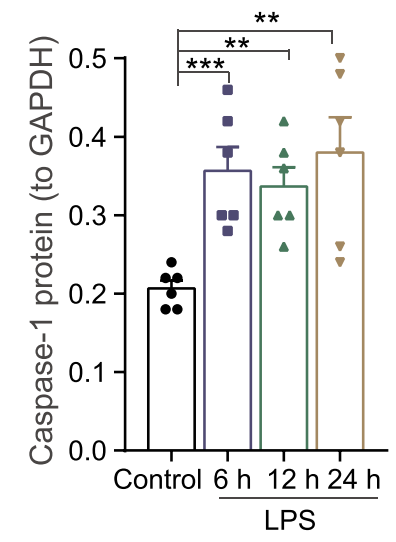

Figure 2 Effect of LPS on the expression NLRP3/caspase-I and pro-inflammatory cytokines in the hippocampus of rats. (A) The qRT-PCR result of the mRNA expression of NLRP3, caspase-I, IL-I $\beta$ and IL-I8. (B) ELISA assay of IL-I $\beta$ and IL-I8 protein. (C) Representative Western blot bands and densitometric analysis of (D) NLRP3 and (E) caspase-I. GAPDH was used as an internal control. Data were presented as mean \pm SEM. $\mathrm{n}=6$ per group. $* * p<0.01, * * * p<0.001$.

Abbreviation: LPS, lipopolysaccharide.

heatmap was shown in Figure 3C. Compared with the control group, miR-138-5p was significantly downregulated in the model group (Figure 3D, $p<0.05$ ). To elucidate the role of NLRP3 and miR-138-5p in the LPS-induced neuroinflammation, we measured the miR-138-5p mRNA expression. We found it was downregulated at $6 \mathrm{~h}, 12 \mathrm{~h}$ and $24 \mathrm{~h}$ after LPS (Figure 3E, $\mathrm{p}<0.001)$, with no significance between different time points $(6 \mathrm{~h}, 12 \mathrm{~h}$ and $24 \mathrm{~h})(p>0.05)$. These results indicate that LPS downregulated miR-138-5p expression in the rat hippocampus.

\section{NLRP3 is a Target Gene of MiR-138-5p in Rat}

Subsequently, we predicted a potential binding site between miR-138-5p and the complementary regions 3 'UTR of NLRP3 gene, with a low minimum free energy (mfe, $-23.7 \mathrm{kcal} / \mathrm{mol}$ ) calculated by the RNAhybrid database (Figure 3F-G). A dual-luciferase reporter assay further confirmed the relationship between miR138-5p and the 3'-UTR of NLRP3. The results showed that co-transfection of cells with NLRP3-3'-UTR-WT and the miR-138-5p mimic resulted in significantly decreased luciferase activity compared with the miR138-5p mimic NC construct ( $\mathrm{p}<0.01$, Figure $3 \mathrm{H})$. However, when the NLRP3-3'-UTR-MUT was cotransfected, no significant difference was found in the luciferase activity between the miR-138-5p mimic and miR-138-5p mimic NC construct groups $(\mathrm{p}>0.05$, Figure $3 \mathrm{H})$. It indicates the specific interaction between miR-138-5p and the 3'-UTR sites of NLRP3, in which NLPR3 is a direct target of miR-138-5p.

\section{Low Expression of MiR-138-5p Was Found in RM Microglial Cells Stimulated by LPS}

Next, we explored the role of miR-138-5p in the neuroinflammation of RM microglial cells by LPS 
A

\begin{tabular}{|c|c|c|c|}
\hline Gene Symbol & $\begin{array}{l}\text { Target } \\
\text { Rank }\end{array}$ & $\begin{array}{l}\text { Target } \\
\text { Score }\end{array}$ & miRNA name \\
\hline NLRP3 & 1 & 79 & rno-miR-138-5p \\
\hline NLRP3 & 2 & 57 & rno-miR-325-5p \\
\hline NLRP3 & 3 & 50 & rno-miR-370-5p \\
\hline
\end{tabular}

C

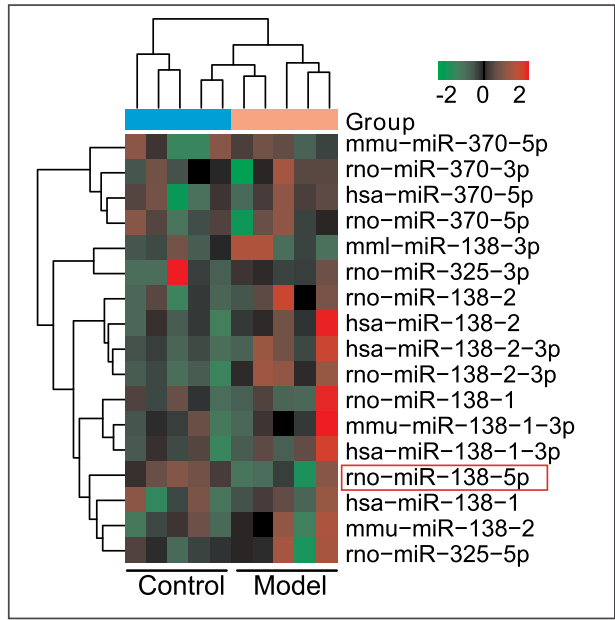

B

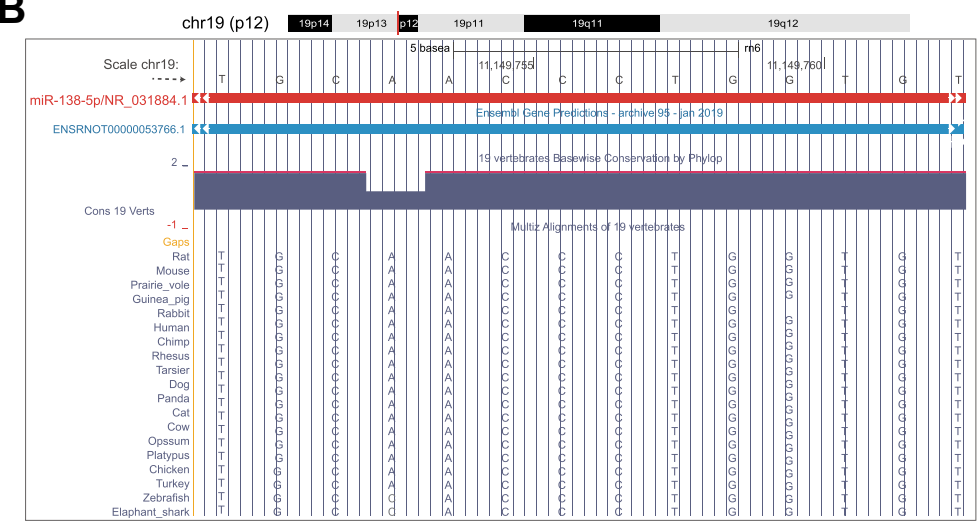

D

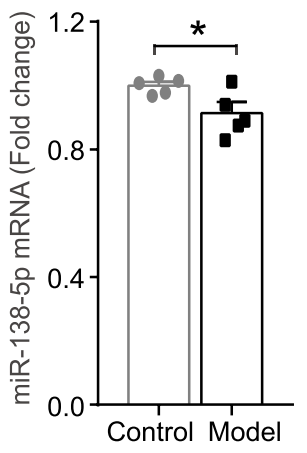

E

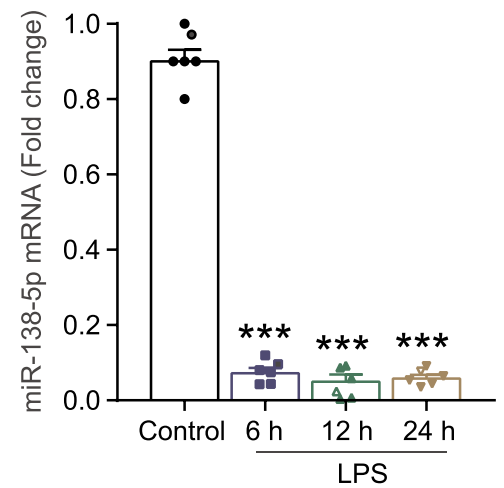

H

H miR-138-5p mimic NC

F [45940137\

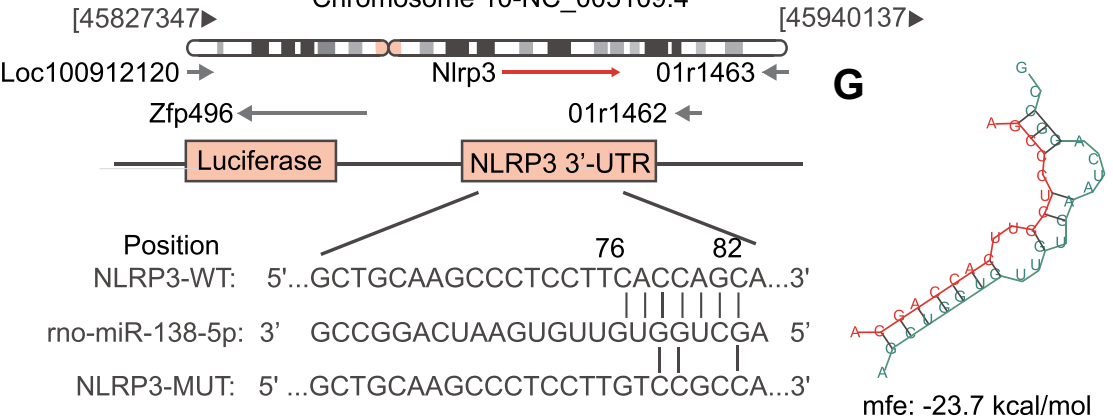

Figure 3 The expression of miR-138-5p in the hippocampus of LPS-treated rats. (A) Three miRNAs that could bind to NLRP3 in rats predicted by miRDB software. (B) The basic information of chromosome location, base sequence and species conservativeness of miR-I38-5p obtained from UCSC Genome Bioinformatics website. (C) Heatmap for the three predicted miRNAs expression and (D) relative miR-138-5p level in the hippocampal of the cognitive impairment animal model and the control group ( $n=5$ pre group) from NCBI GEO database. (E) The qRT-PCR result of miR-138-5p mRNA expression in the LPS-treated rat hippocampus ( $n=6$ pre group). (F) The predicted binding sites and $(\mathbf{G})$ mfe between miR-I38-5p and the 3'-UTR of NLRP3. (H) A firefly luciferase reporter containing NLRP3-WT/MUT with miR-I38-5p mimic $\mathrm{NC} /$ mimic $\left(\mathrm{n}=3\right.$ pre group). Data were presented as mean $\pm \mathrm{SEM}$. ${ }^{*} p<0.05, * * p<0.01$.

Abbreviations: GEO, Gene Expression Omnibus; LPS, lipopolysaccharide; NCBI, National Center for Biotechnology Information; GEO, Gene Expression Omnibus; mfe, minimum free energy; WT, wild-type; MUT, mutant; NC, negative control; ns, not significant.

stimulation in vitro. As shown in Figure 4A, miR-138$5 \mathrm{p}$ was significantly downregulated in the LPS group at $6 \mathrm{~h}, 12 \mathrm{~h}$ and $24 \mathrm{~h}$ than those in the control group ( $p<$ 0.01-0.001). The mRNA expression of NLRP3, caspase1 and pro-inflammatory cytokines (IL-1 $\beta$ and IL-18) was detected by qRT-PCR, which showed a higher mRNA level in the LPS-treated group compared with the control ( $p<0.05-0.001$, Figure 4B). Meanwhile, the results of ELISA and Western blot analysis showed that the protein of IL-1 $\beta$, IL-18, NLRP3, and caspase-1 was significantly increased in RM cells stimulated by LPS $(p<0.05-0.001$, Figure 4C-F). Additionally, Hoechst 3342 staining showed that LPS induced cell apoptosis significantly compared with the control group 
A

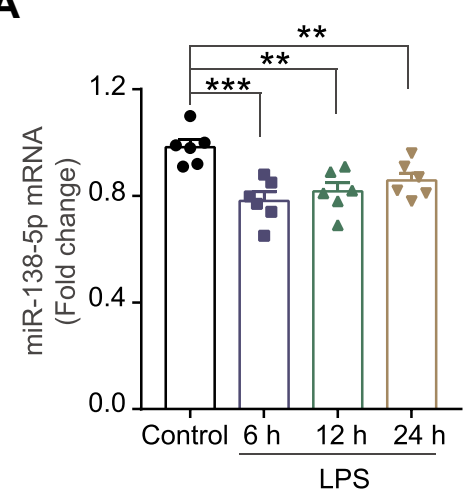

D

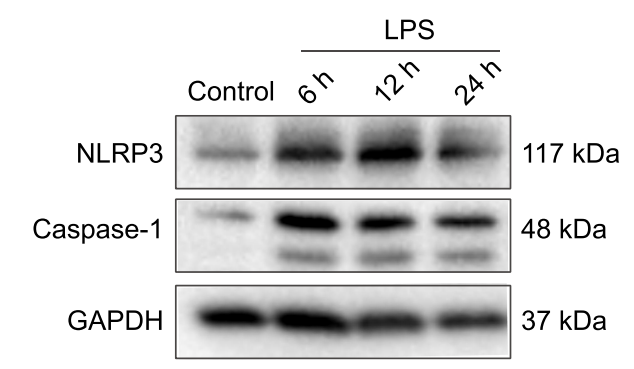

G

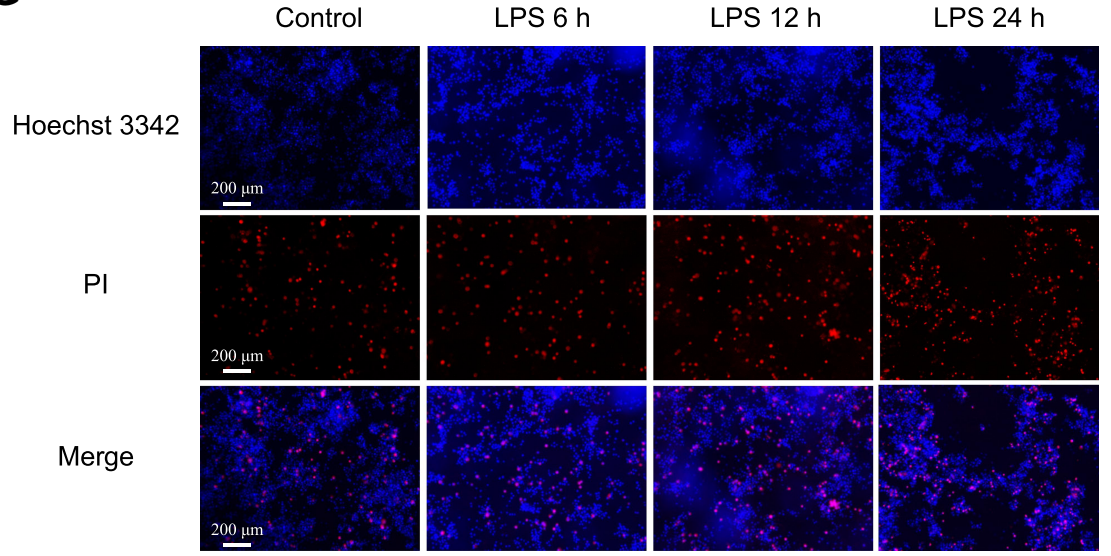

E

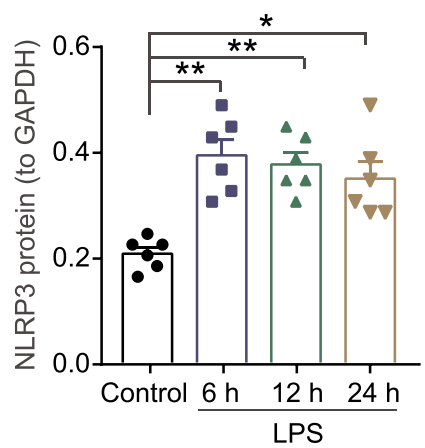

C

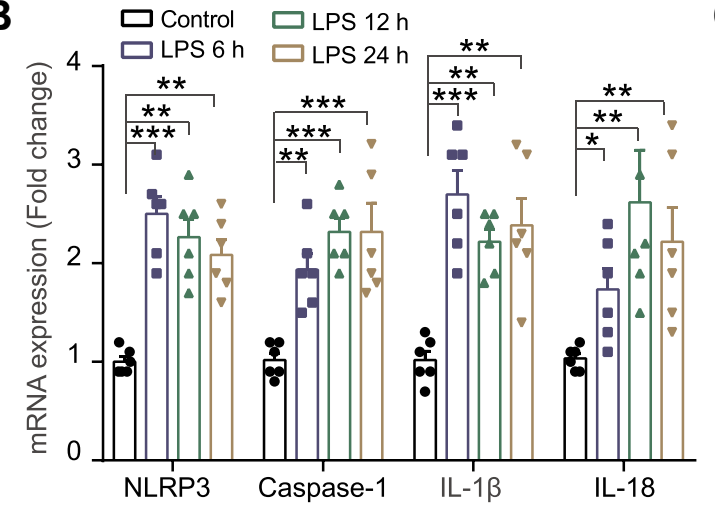

F
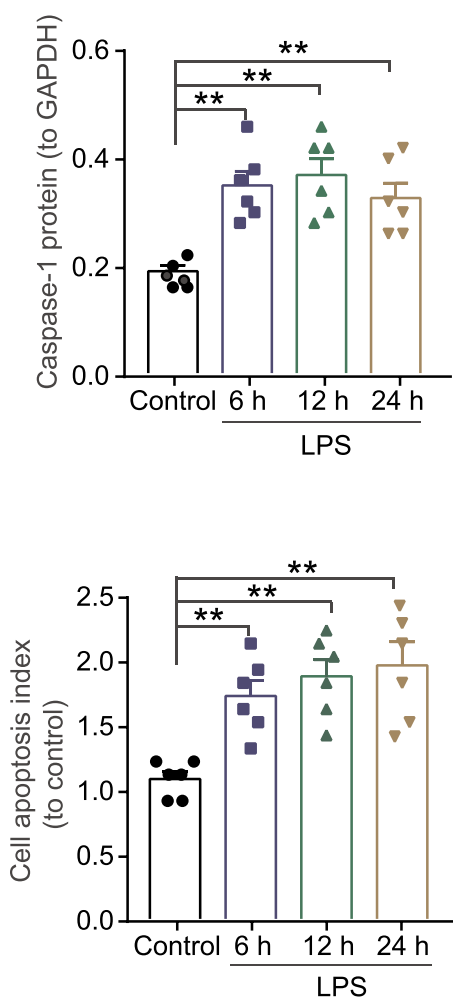

Figure 4 Effect of LPS on the expression of miR-I38-5p and NLRP3/caspase-I in RM microglial cells. The qRT-PCR result of (A) miR-I38-5p, (B) NLRP3, caspase-I, IL-I $\beta$ and IL- 18 mRNA expression in RM cells at $6 \mathrm{~h}, 12 \mathrm{~h}$ and $24 \mathrm{~h}$ after LPS. (C) ELISA assay of IL-I $\beta$ and IL- 18 protein expression. (D) Representative Western blot bands and densitometric analysis of (E) NLRP3 and (F) caspase- I in RM cells at $6 \mathrm{~h}, 12 \mathrm{~h}$ and $24 \mathrm{~h}$ after LPS. (G) Hoechst $33342 / \mathrm{PI}$ dual staining assays and counted of cell apoptosis in RM cells at $6 \mathrm{~h}, \mathrm{I} 2 \mathrm{~h}$ and $24 \mathrm{~h}$ after LPS. GAPDH was used as an internal control. Data were presented as mean $\pm \mathrm{SEM}$. $\mathrm{n}=6 \mathrm{per}$ group. ${ }^{*} p<0.05$, **p $<0.0 \mathrm{I}, * * * p<0.00 \mathrm{I}$. Abbreviation: LPS, lipopolysaccharide; RM, rat microglia; PI, propidium iodide.

at $6 \mathrm{~h}, 12 \mathrm{~h}$ and $24 \mathrm{~h}(p<0.01$, Figure $4 \mathrm{G})$. And there was no significant difference between groups at different time points after LPS $(p>0.05)$. Thus, RM cells were treated with $1 \mu \mathrm{g} / \mathrm{mL}$ for $6 \mathrm{~h}$ in vitro transfection experiment. Taken together, LPS decreases the miR138-5p expression, which promotes the expression of NLRP3/caspase-1 and cell apoptosis, resulting in neuroinflammation of RM microglial cells.

\section{MiR-I38-5p Downregulates the}

\section{LPS-Induced Inflammatory Response in RM Microglial Cells}

To clarify whether miR-138-5p could regulate the activation of NLRP3/caspase-1 pathway, we further evaluated the effects of miR-138-5p inhibitor or mimic on the NLRP3 activation-modulated inflammatory response in 

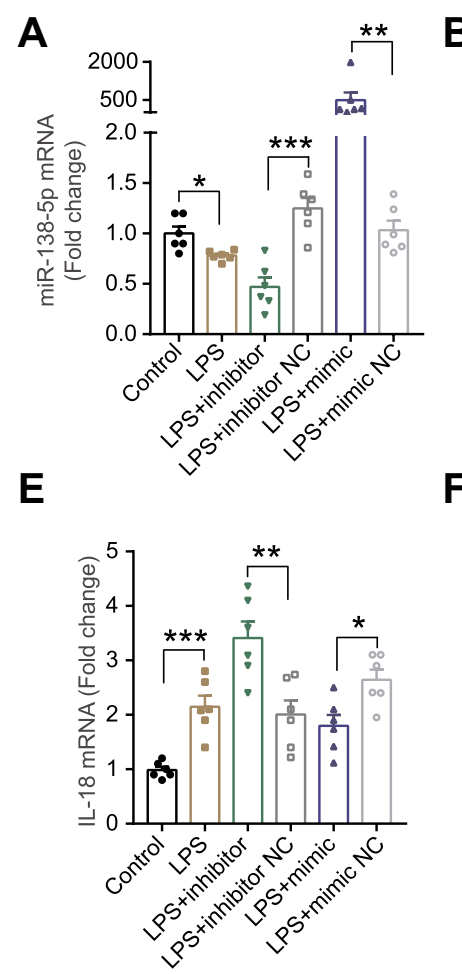

I

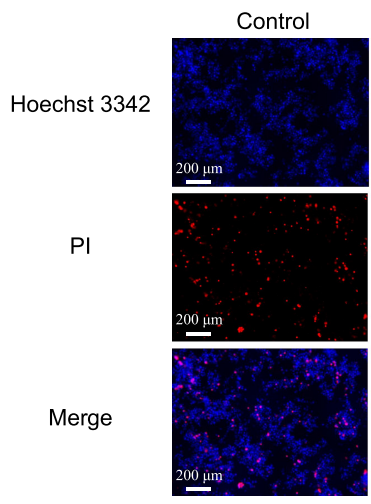

$\mathbf{J}$

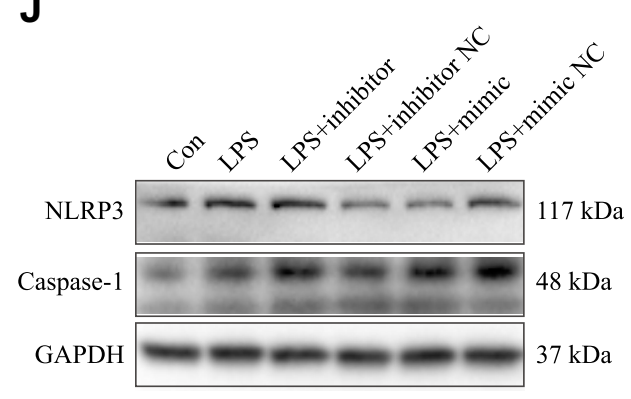

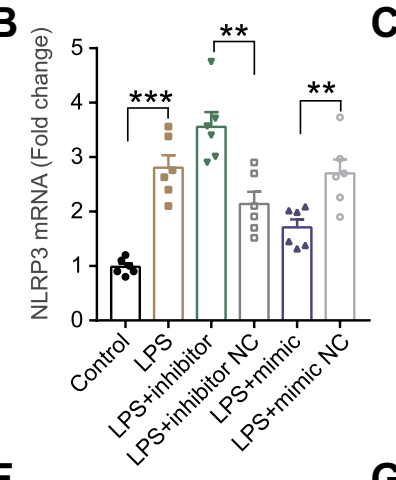
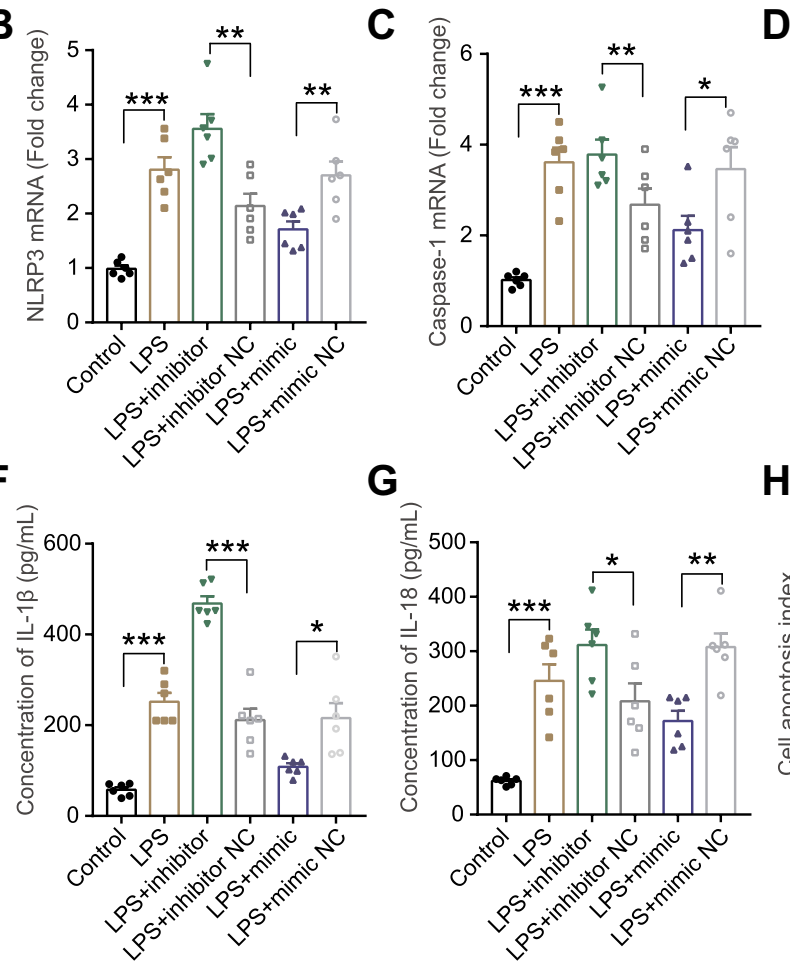

H
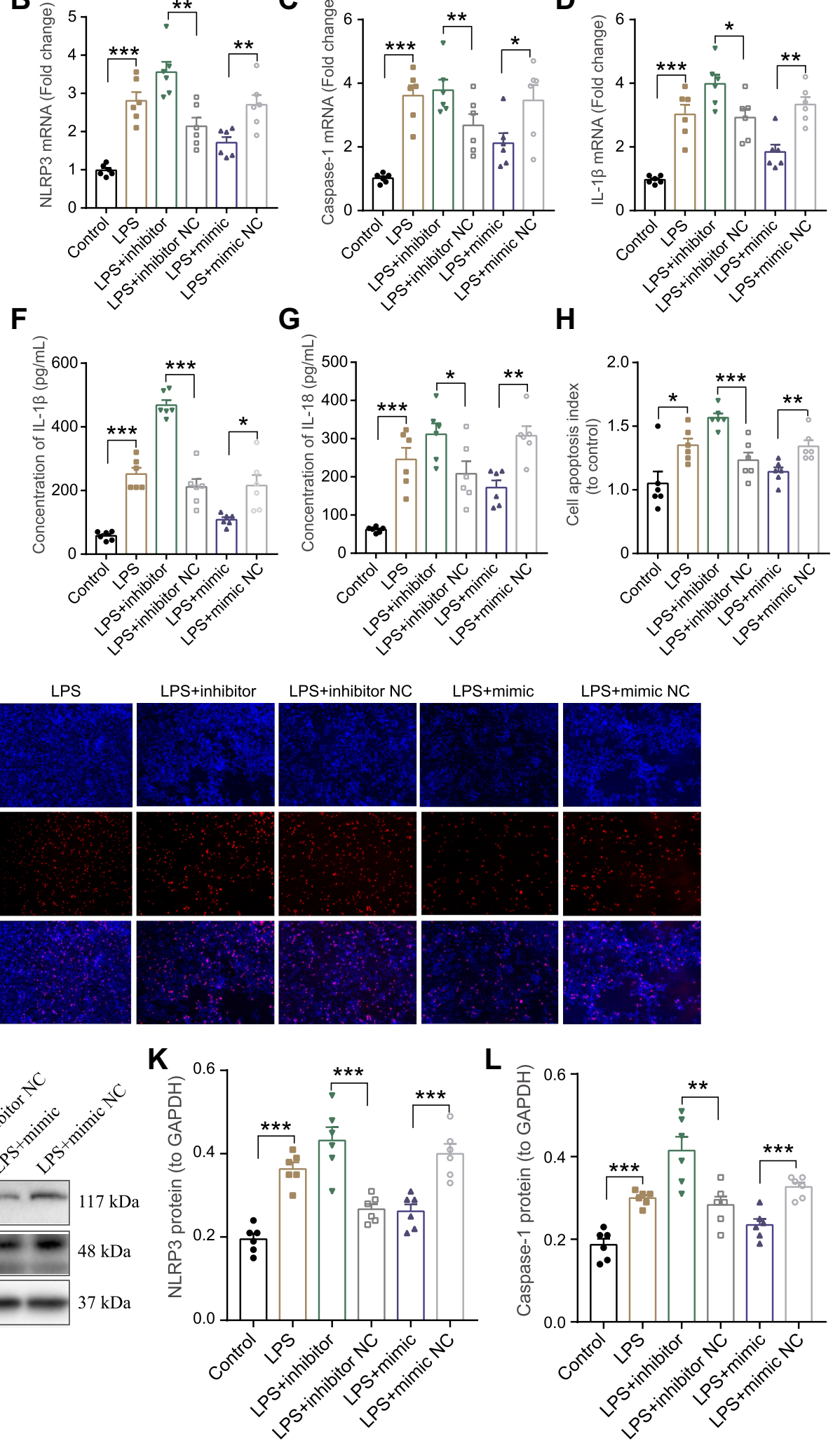

Figure 5 Effect miR-138-5p on LPS-induced inflammatory response in RM microglial cells. The qRT-PCR result of (A) miR-I38-5p, (B) NLRP3, (C) caspase-I, (D) IL-I $\beta$, and (E) IL18 mRNA expression in LPS-treated RM microglial cells after pretreatment with miR-I38-5p inhibitor or mimic. (F-G) ELISA result of IL-I $\beta$ and IL-I8 protein expression after pretreatment with miR-138-5p inhibitor or mimic in LPS-treated RM microglial cells. (H-I) Hoechst 33342/PI dual staining assays and counted of cell apoptosis in LPS-treated RM microglial cells after pretreatment with miR- I38-5p inhibitor or mimic. (J) Representative Western blot bands and densitometric analysis of (K) NLRP3 and (L) caspase-I after pretreatment with miR-138-5p inhibitor or mimic in LPS-treated RM microglial cells. Data were presented as mean \pm SEM. $n=6$ per group. $* p<0.05$, ** $p<0.0$ I, $* * * p<0.001$. Abbreviations: LPS, lipopolysaccharide; RM, rat microglia; NC, negative control; PI, propidium iodide. 
RM cells by a transfection experiment. The transfection efficiency of miR-138-5p inhibitor or mimic was confirmed by qRT-PCR. As shown in Figure 5A, transfection with miR-138-5p inhibitor for $72 \mathrm{~h}$ significantly decreased miR-138-5p expression compared to transfection with the inhibitor NC $(p<0.001)$, while mimic significantly increased its expression $(p<0.01)$. The NLRP3/caspase1 mRNA expression was significantly upregulated in LPS + inhibitor group compared to the LPS + inhibitor NC group, whereas significantly downregulated in LPS + mimic group compared to the LPS + mimic NC group $(p<0.05$, Figure 5B-C). Similarly, transfection with miR$138-5 \mathrm{p}$ inhibitor increased the mRNA and protein expression of pro-inflammatory cytokines (IL-1 $\beta$ and IL-18), while mimic decreased the expression of these proinflammatory factors $(p<0.05-0.001$, Figure 5D-G). Hoechst 3342 staining showed that LPS-induced cell apoptosis was enhanced by miR-138-5p inhibitor, and weaken by miR-138-5p mimic $(p<0.05-0.001$, Figure $5 \mathrm{H}-\mathrm{I})$. Furthermore, the miR-138-5p inhibitor significantly increased the NLRP3/caspase-1 protein expression in the LPS model of RM cells, whereas miR-138-5p mimic decreased its expression $(p<0.01-0.001$, Figure 5J-L). These results suggest that miR-138-5p could downregulate neuroinflammation induced by LPS in RM microglial cells.

\section{MiR-138-5p Ameliorates LPS-Induced Cognitive Impairment of Rats in MWM} Test and Y-Maze Test

The above results indicated that inflammation response showed no significance between different time points $(6 \mathrm{~h}$, $12 \mathrm{~h}$ and $24 \mathrm{~h}$ ) after LPS injection (50 $\mu \mathrm{g})$, and rats showed a severe decline in spatial learning and memory on day 1 after LPS. Thus, for the next vivo transfection experiment, rats were treated with LPS $(50 \mu \mathrm{g})$ for $24 \mathrm{~h}$. To further evaluate the effects of miR-138-5p on LPS-induced memory impairment, we administrate miR-138-5p antagomir/agomir into rats before LPS injection. The experimental outline was summarized in Figure 6A. Similarly, compared with the control group, the LPS-treated rats showed significant cognition impairment in MWM test and Y-maze test. Moreover, pretreatment with miR-138-5p antagomir in LPS-induced rats showed longer escape latency and less number of crossing the platform compared with the LPS + antagomir NC group. On the contrary, miR-138-5p agomir could shorten escape latency and increase the number of crossing the platform in LPS-treated rats $(p<0.05$, Figure 6B-D). No significant difference was observed in the time spent in the target quadrant between different groups ( $p>0.05$, Figure $6 \mathrm{E})$. Also, the result of Y-maze test showed that the alternation was significantly decreased in the LPS + antagomir group, while significantly increased in the LPS + agomir group compared with $\mathrm{NC}$ rats $(p<0.05$, Figure $6 \mathrm{~F})$. However, no significant difference in the number of arm entries was observed in different groups $(p>0.05$, Figure $6 \mathrm{G})$. These findings demonstrate that miR-138-5p ameliorates LPS-induced cognitive decline in rats.

\section{MiR-I 38-5p Suppresses the Expression of NLRP3/Caspase-I and Pro-Inflammatory Cytokines in the Hippocampus of LPS-Treated Rats}

Neuronal morphology of the hippocampal tissue was observed by H\&E staining (Figure 7A). The hippocampal nerve cells in the control group were regularly arranged, with bright nucleoli and clear nuclei. LPS injection significantly reduced the number of normal neural cells, resulting in hippocampal neurons being irregularly arranged, nuclear retraction and tissue edema, as indicated by arrows. Furthermore, pretreatment with miR-138-5p antagomir in LPS-induced rats showed more karyopyknosis and edema, while the hippocampus neural cells in miR138-5p agomir group were arranged more densely and neatly ( $p<0.05-0.01$, Figure 7B).

Next, we examine the effect of miR-138-5p on hippocampal inflammation in rats with cognitive impairment. As shown in Figure 7C, LPS downregulated hippocampal miR-138-5p expression, and this decrease would be further reduced by miR-138-5p antagomir, while increased by miR-138-5p agomir $(p<0.001)$. Furthermore, we found that miR-138-5p antagomir significantly increased mRNA expression of NLRP3, caspase- 1 and IL-1 $\beta$ in the hippocampus $(p<0.05-0.001)$, but not IL-18 $(p>0.05)$; while miR-138-5p agomir decreased the above-mentioned mRNA expression $(p<$ 0.05-0.001, Figure 7D-G). The result of ELISA (Figure $7 \mathrm{H}-\mathrm{I}$ ) and western bolt (Figure 7L-N) showed that miR$138-5 \mathrm{p}$ antagomir significantly increased the protein levels of IL-1 $\beta$, IL-18, NLRP3 and caspase-1 compared to the LPS + antagomir NC rats $(p<0.05-0.001)$. On the contrary, miR-138-5p agomir significantly decreased the above-mentioned protein expression in the hippocampus of rats $(p<0.01-0.001)$. Besides, 
A Environmental adaptation

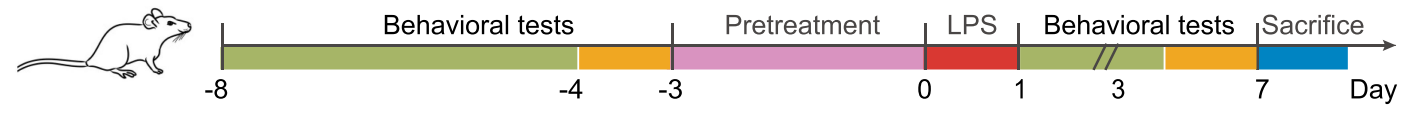

Acquisition trail (MWM test)

Probe trial (MWM test), Y-maze

icv LPS

icv miR-138-5p antagomir/agomir
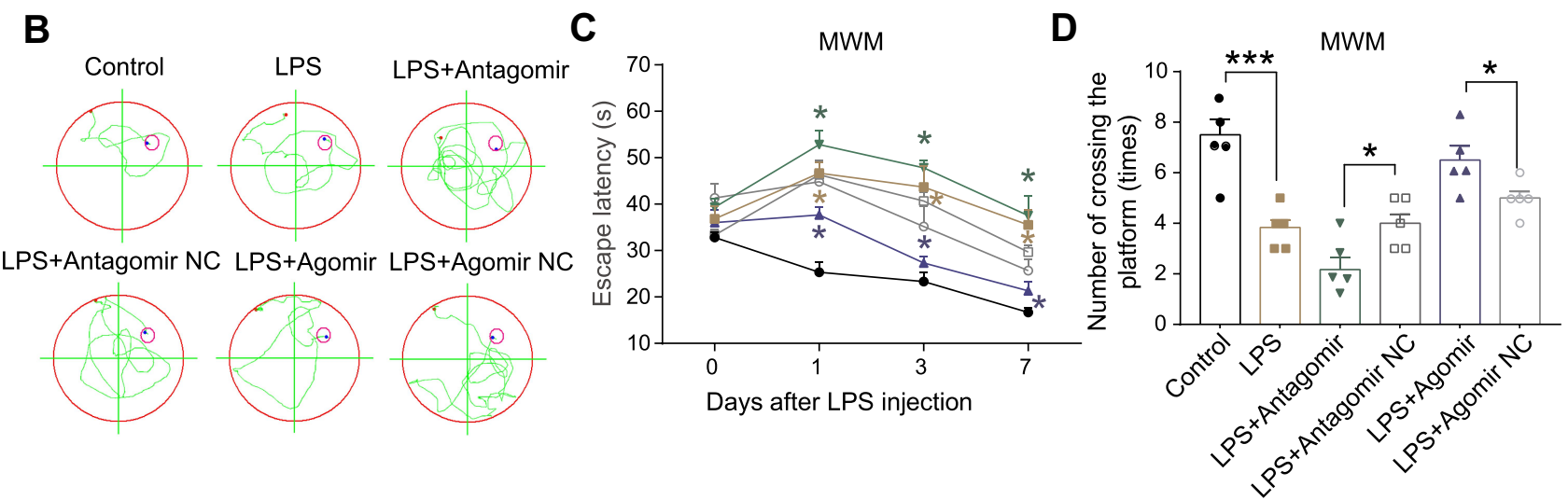

$\rightarrow$ - Control $\rightarrow$ - LPS $\quad \rightarrow$ LPS+Antagomir $\quad \rightarrow$ LPS+Antagomir NC

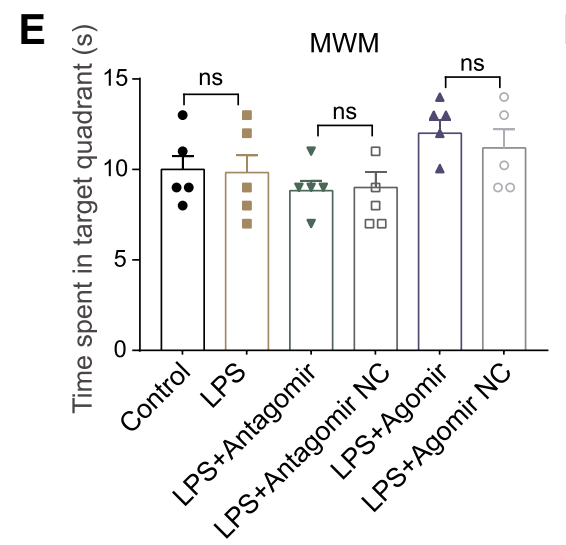

$\mathbf{F}$
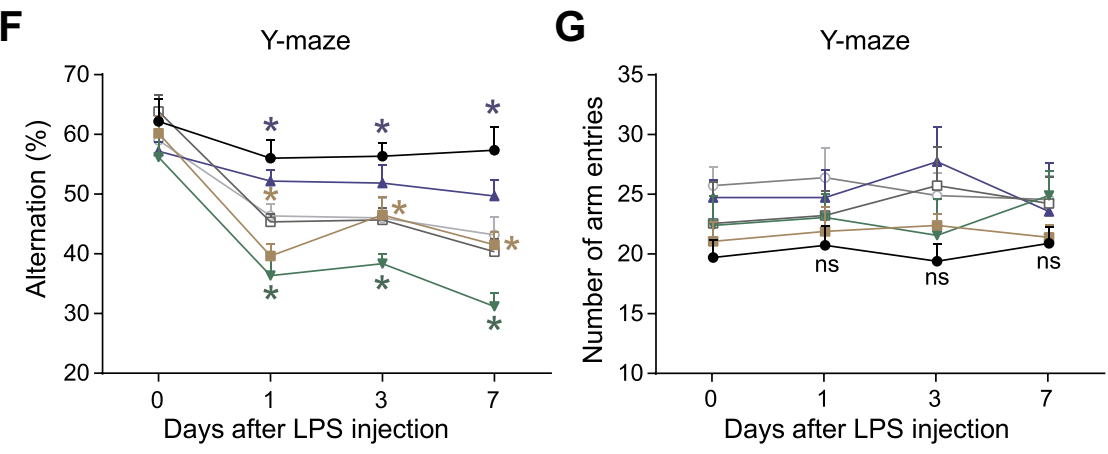

Figure 6 Effect of miR-138-5p on LPS-induced cognitive impairment behaviors of rats in MWM Test and Y-maze Test. (A) Experimental protocol and timeline. (B) Representative swimming path on day 7 after LPS injection in MWM test. Effect of miR-138-5p antagomir and agomir on (C) escape latency, (D) number of crossing the platform, and (E) time spent in the target quadrant in MWM test. Effect of miR-I38-5p antagomir and agomir on $(\mathbf{F})$ alternation and $(\mathbf{G})$ number of arm entries in $Y$-maze test. Data were presented as mean \pm SEM. $n=5$ per group. ${ }^{*} p<0.05,{ }^{* *} p<0.001$.

Abbreviations: icv, intracerebroventricular; LPS, lipopolysaccharide; RM, rat microglia; NC, negative control; MWM, Morris water-maze; ns, not significant.

immunohistochemistry indicated that LPS-treated rats showed a larger number of NLRP3 positive cells in the hippocampus than the control ones, and miR-138$5 \mathrm{p}$ antagomir enhanced this effect, while agomir repressed this effect $(p<0.05-0.01$, Figure $7 \mathrm{~J}-\mathrm{K})$, as indicated by arrows. These results demonstrate that miR-138-5p could suppress the expression of hippocampal NLRP3/caspase-1 and pro-inflammatory cytokines of LPS-treated rats.

\section{MiR-I38-5p Inhibits Microglial Activation in the Hippocampus of LPS-Treated Rats}

Microglial activation is critical for hippocampal neuroinflammation in cognitive impairment, so we used immunohistochemistry to detect the number of positive Iba-1 (a microglia marker) cells. As shown in Figure 8A-B, we found that numerous Iba- 1 was positively stained in the hippocampus evoked by LPS compared to the control 

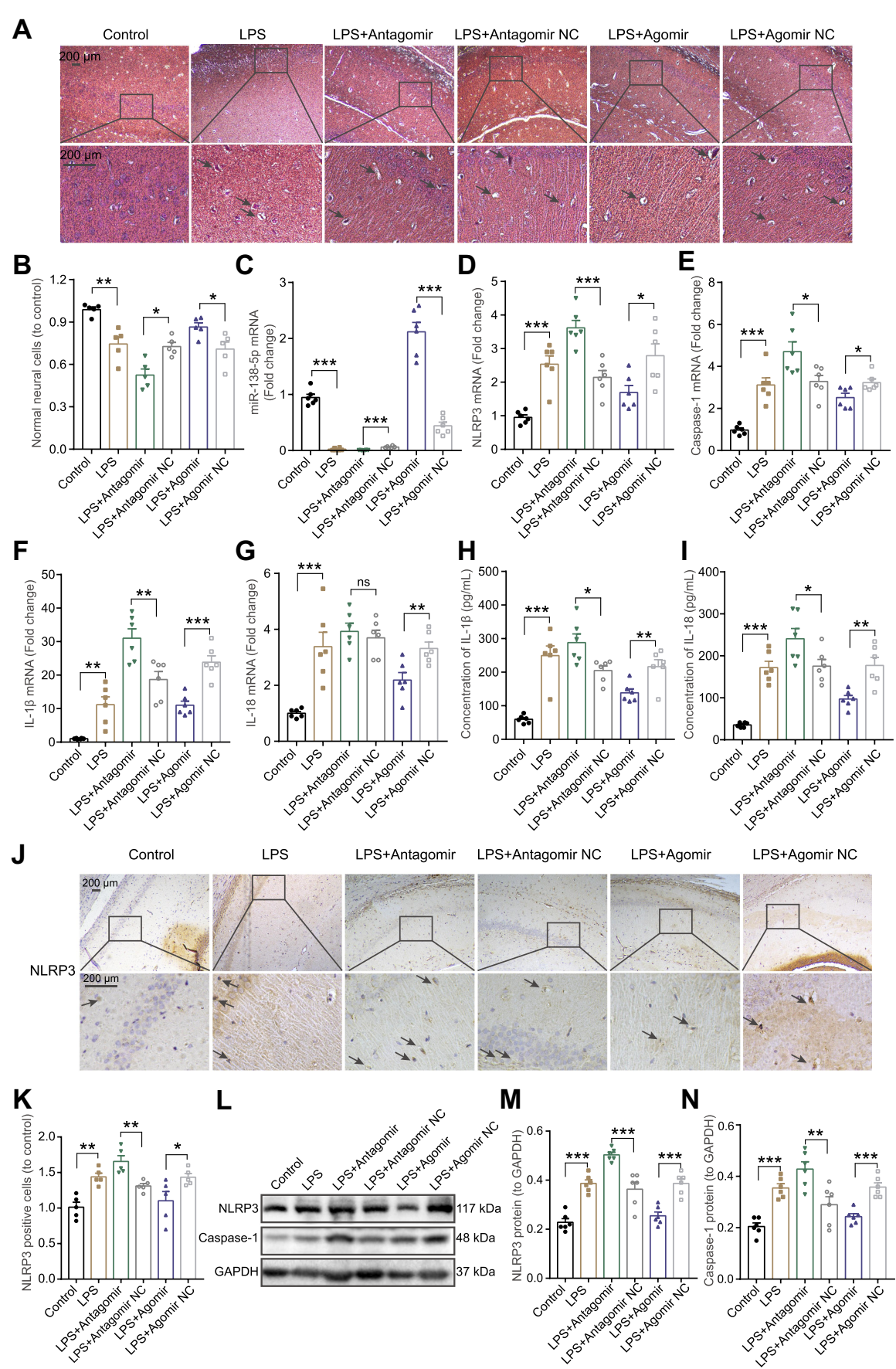

Figure 7 Effect of miR-138-5p on the expression of NLRP3/caspase-I and pro-inflammatory cytokines in the hippocampus of LPS-treated rats. (A) Representative H\&E staining images and (B) number of neural cells in the hippocampus of rats. The arrow points to irregularly arranged, nuclear retraction and tissue edema. The qRT-PCR result of (C) miR-I38-5p, (D) NLRP3, (E) caspase-I, (F) IL-I $\beta$ and (G) IL- 18 mRNA expression after miR-I38-5p antagomir or agomir pretreatment in LPS-treated rats ( $\mathrm{n}=6 \mathrm{per}$ group). ELISA result of $(\mathbf{H})$ IL-I $\beta$ and $(\mathbf{I})$ IL- 18 protein expression after pretreatment with miR-I38-5p antagomir or agomir in LPS-treated rats ( $\mathrm{n}=6$ per group). (J-K) Representative immunohistochemistry staining images and count of NLRP3 positive cells in LPS-treated rats after pretreatment with miR-I38-5p antagomir or agomir ( $\mathrm{n}=$ 5 per group). The arrow points to NLRP3 positive cells. (L) Representative Western blot bands and densitometric analysis of (M) NLRP3 and (N) caspase-I after pretreatment with miR-I38-5p antagomir or agomir in LPS-treated rats $(\mathrm{n}=6$ per group). Data were presented as mean \pm SEM. $* p<0.05$, $* * p<0.0 \mathrm{I}$, $* * * p<0.00 \mathrm{I}$.

Abbreviations: H\&E, Hematoxylin and eosin; LPS, lipopolysaccharide; NC, negative control; ns, not significant.

group. After pretreatment with miR-138-5p antagomir, the Iba-1 positive cells were significantly increased, while significantly decreased in the miR-138-5p agomir group $(p<$ $0.05-0.01)$, as indicated by arrows. Furthermore, we detected the expression of mRNA and protein of hippocampal Ibal of rats, which were upregulated in the hippocampus of LPS rats compared to that of the control rats. Pretreatment miR-138$5 p$ antagomir increased hippocampal Ibal expression in rats, 


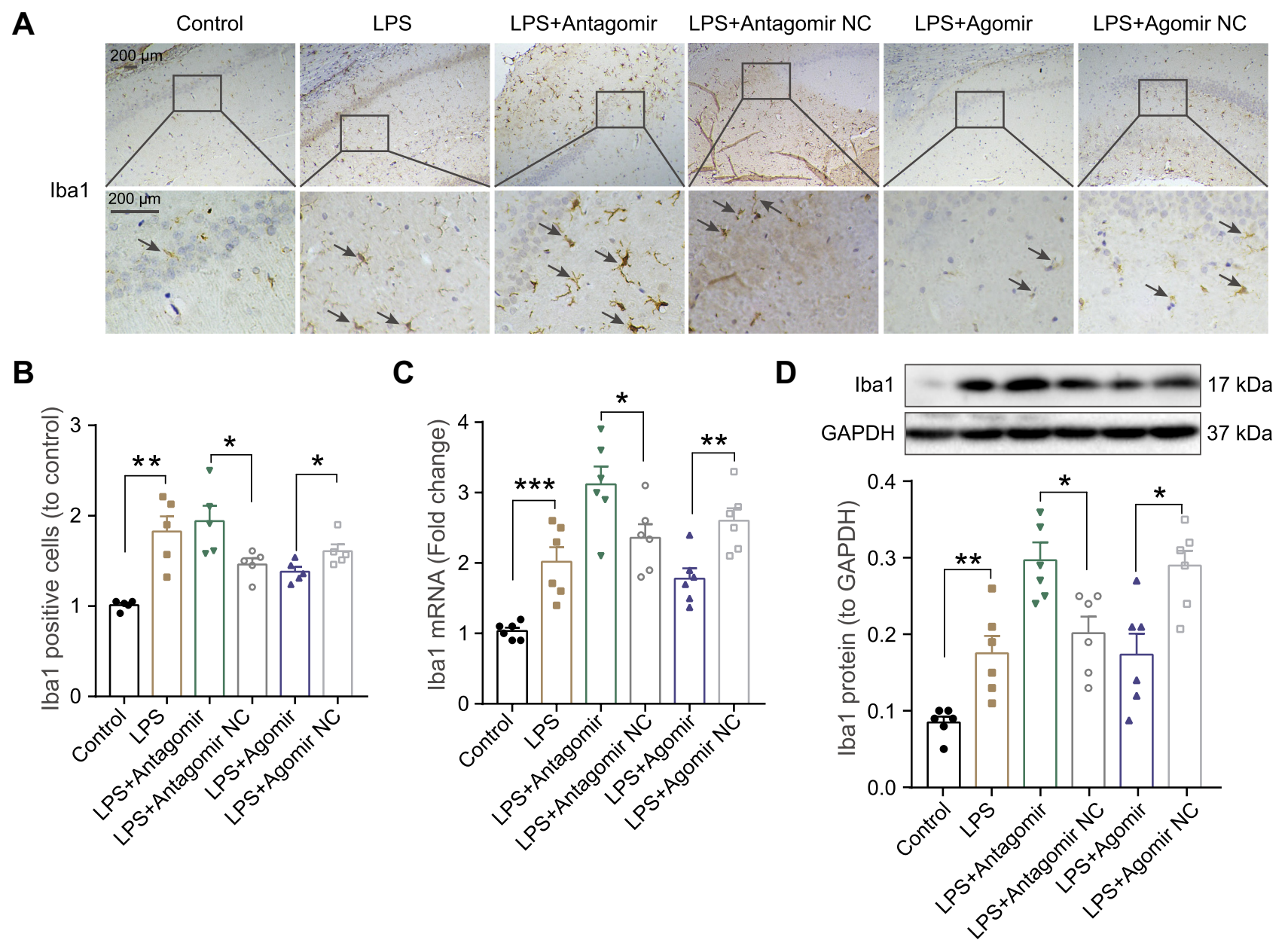

Figure 8 Effect miR-138-5p on the microglial activation in the hippocampus of LPS-treated rats. (A) Representative immunohistochemistry staining images and (B) count of Ibal positive cells in LPS-treated rats after pretreatment with miR-I38-5p antagomir or agomir ( $n=5$ per group). The arrow points to lbal positive cell. (C) The qRT-PCR result of Ibal mRNA expression, (D) representative Western blot bands and densitometric analysis of Ibal protein in LPS-treated rats after pretreatment with miR-I38-5p antagomir or agomir. GAPDH was used as an internal control $\left(n=6\right.$ per group). Data were presented as mean \pm SEM. $*_{p}<0.05, * * p<0.01$, *** $p<0.00 \mathrm{I}$.

Abbreviations: LPS, lipopolysaccharide; NC, negative control.

while miR-138-5p agomir decreased Iba1 expression compared to LPS + antagomir/agomir NC rats $(p<0.05-0.001$, Figure $8 \mathrm{C}-\mathrm{D})$. Collectively, these findings show that miR$138-5 p$ negatively regulates microglial activation in a rat model of cognitive impairment.

\section{Discussion}

In this study, we evaluated the potential mechanism underlying miR-138-5p/NLRP3/caspase-1 axis on LPSinduced hippocampal neuroinflammation and cognitive impairment in rats. First, we found that LPS decreased miR-138-5p expression in rats hippocampus and RM microglial cells, increased hippocampal neuroinflammation as demonstrated by upregulated expression of NLRP3/caspase-1 and pro-inflammatory cytokines (IL$1 \beta$ and IL-18), as well as activated microglia. Moreover, our results reveal that NLRP3 is the direct target gene of miR-138-5p; the supplement of miR-138-5p by pretreatment with miR-138-5p mimic/agomir could ameliorate neuroinflammation, hippocampus-dependent learning and memory decline in LPS-treated rats. Taken together, the results demonstrate that miR-138-5p could negatively regulate LPS-induced hippocampal neuroinflammation and cognitive impairment through inhibiting the NLRP3/caspase-1 signaling pathway activation (Figure 9).

The precise pathogenesis of the neurological disorder is complex and remains elusive, resulting in the lacking of effective treatments and potential therapeutic molecules. The CNS inflammation, in particular hippocampal neuroinflammation, is associated with hippocampal neurogenesis and cognitive impairment-related diseases, such as 


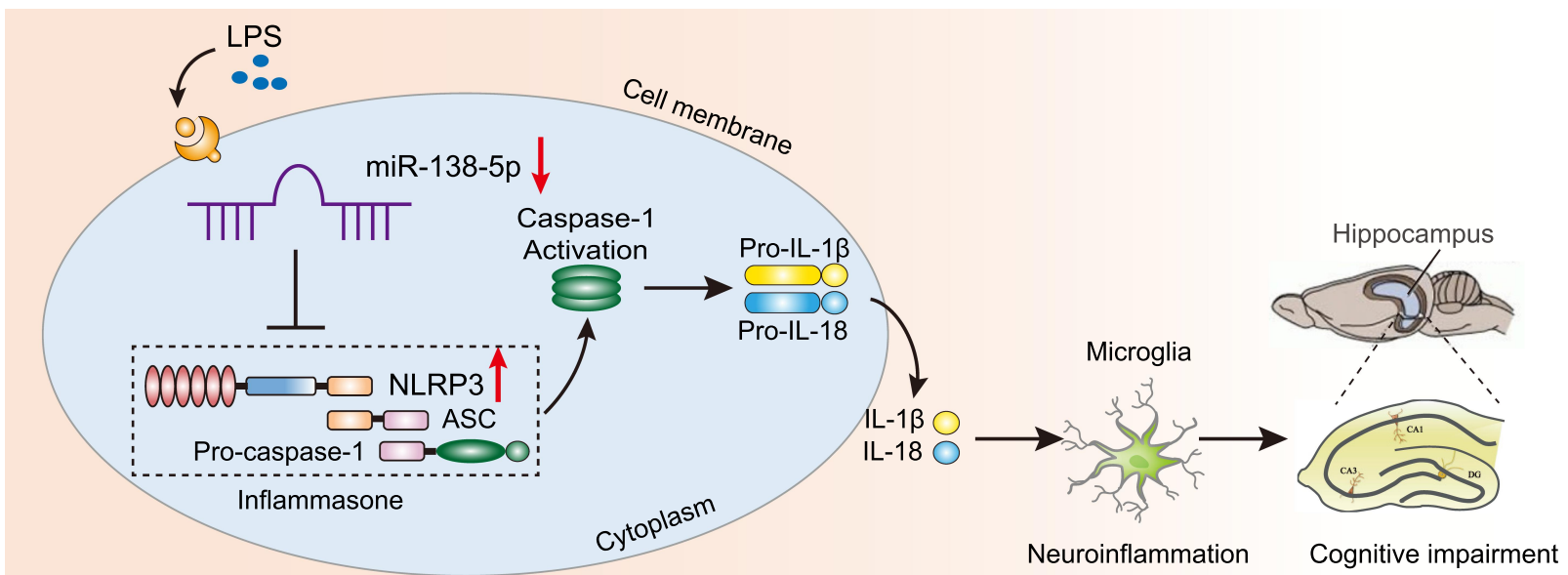

Figure 9 Schematic diagram description of the mechanism underlying miR-138-5p/NLRP3/caspase-I axis in LPS-induced hippocampal neuroinflammation and cognitive impairment. LPS downregulates the miR-138-5p expression, then activates the NLRP3 inflammasome results in caspase-I activation, which cleaves pro-IL-I $\beta$ and pro-IL- 18 into IL-I $\beta$ and IL-18. Consequently, hippocampal neuroinflammation level was increased and induced cognitive impairment.

Abbreviations: LPS, lipopolysaccharide; ASC, apoptosis-associated speck-like protein.

PD, AD and PND. ${ }^{3-5}$ Studies have indicated that LPSinduced neuroinflammation models are valid for deciphering the mechanism underlying neurodegeneration and cognitive impairment. ${ }^{39-42}$ The MWM test and Y-maze test, the most common behavioral tools in neuroscience, were used as reliable tests that reflect hippocampaldependent learning and memory in laboratory animals. $^{33,35}$ Therefore, we established an LPS-induced neuroinflammation model to study the cognitive dysfunction, while selected the MWM test and Y-maze test to evaluate the cognitive function in this study. Consistent with previous studies, ${ }^{39-41}$ our study found that icv injection of LPS produced significant cognitive decline behaviors both in MWM test and Y-maze test, suggesting our cognitive impairment model induced by LPS was successful.

Of the NLRs family, the NLRP3 inflammasome is the most typical and widely studied. It triggers caspase-1 activation, which cleaves inactive cytokine precursors (pro-IL-1 $\beta$ and pro-IL-18) into pro-inflammatory cytokines (IL-1 $\beta$ and IL-18). These pro-inflammatory cytokines have been reported involved in some inflammatory diseases and neurological disorders. ${ }^{43}$ Previous studies have shown that NLRP3 was upregulated in the hippocampus of cognitive impairment models induced by anesthesia and surgery. ${ }^{19-21}$ Similarly, our study further revealed that LPS enhanced the expression of NLRP3/caspase-1 and pro-inflammatory cytokines (IL-1 $\beta$ and IL-18) in the hippocampus of rats and RM microglial cells. Thus, our findings highlighted that the NLRP3 inflammasome might be a potential target for cognitive impairment, and in-depth research on the activation mechanism of the NLRP3 inflammasome seems particularly critical.

Differentially expressed miRNAs in the CNS play essential roles in various diseases associated with cognitive impairment. ${ }^{10,14,44-46}$ Accumulating evidence indicated the vital role of miR-138-5p in the development and homeostasis of the immune system. ${ }^{23-28}$ In the majority, miR-138-5p seems to act as a tumor-suppressor miRNA by targeting specific oncogenic genes and is also being deregulated in cancer cells, such as glioma, ${ }^{23}$ non-small cell lung cancer, ${ }^{24}$ ovarian $^{47}$ and prostate cancer. ${ }^{25}$ In the present study, low expression of miR-138-5p was found in the rat hippocampus of the cognitive impairment model and RM microglial cells stimulated with LPS. This result was consistent with the finding that miR-138-5p was downregulated in the hippocampus of contextual fear conditioning memory formation, suggesting its vital role on memory and learning. ${ }^{48}$ Besides, this result was supported by a miRNAs profiling based on a cohort of $41 \mathrm{AD}$ patients and 23 controls, which the miR$138-5 p$ relative expression was a 0.62 -fold change in $\mathrm{AD}$ group compared with the control group. ${ }^{49}$

Furthermore, we found that the treatment of miR-138-5p mimic/agomir suppressed the expression of NLRP3, caspase-1, pro-inflammatory cytokines and neuroinflammation of hippocampal neurons, thus improved the learning and memory ability of rats. Also, several studies reported a similar regulation relationship between miRNAs and NLRP3 inflammasome in cognitive disfunction-related disorders. ${ }^{14,44,50-54}$ The expression of miR- $7^{51-53}$ and miR$30 \mathrm{e}^{50}$ were decreased in PD or models, and modulating 
neuroinflammation in the pathogenesis of PD via directly targeting NLRP3 inflammasome, resulting in $\alpha$-synuclein overexpression of dopaminergic neuron. MiR-22 mimic could improve the memory abilities of AD mice by inhibiting the inflammatory factors expression and NLRP3 inflammasome activation. $^{54}$ MiR-124-3p level of microglial exosomes was significantly altered in the brain of repetitive mild traumatic brain injury model, and treatment with miR124-3p could improve the cognitive outcome. ${ }^{44}$ MiR-146a regulates the IRAK1/TRAF6/NF- $\kappa$ B receptor pathway in the PND model, and supplement of miR-146a may be a potential strategy for cognitive function. ${ }^{14}$ Taken together, all the above results demonstrated that NLRP 3 was the target gene of miR-138-5p, and treatment with miR-138-5p may provide a potential protective effect against LPS-induced cognition impairments in neurodegenerative diseases.

There are several limitations in our study. First, we only assessed the relatively short-term cognitive behaviors (7 days) after LPS injection, while long-term cognitive change needs further research. Second, we only studied the role of miR-138-5p on microglial activation, but not other cells involved in immune responses in the CNS, such as astrocytes; and microglial polarization will also be identified. Third, only the cognitive impairment model of LPS-induced inflammation was adopted in this study, while the consistency of the underlying mechanism of miR-138-5p in different cognitive dysfunction models, such as surgery/anesthesia or other neurodegenerative diseases, requires further study. Finally, we only used male rats in this study; a comparison of the effects of miR-138-5p on hippocampal inflammation and cognitive behaviors in female rats remains unexplored.

\section{Conclusions}

In summary, our study for the first time demonstrates that the upregulation of miR-138-5p inhibits hippocampal neuroinflammation, improving the cognitive impairment through suppressing NLRP3/caspase-1 signaling pathway activation in LPS-treated rats. These behavioral and neurochemical improvements suggest that miR-138-5p/ NLRP3/caspase-1 axis may be a promising treatment to improve neurodegenerative disorders.

\section{Abbreviations}

PD, Parkinson's disease; AD, Alzheimer's disease; HD, Huntington's disease; PND, perioperative neurocognitive disorders; LPS, lipopolysaccharide; CNS, central nervous system; NLRs, NOD-like receptors; NLRP3, NLR family pyrin domain-containing-3; ASC, apoptosis-associated speck-like protein; miRNAs, microRNAs; mRNAs, messenger RNAs; SD, Sprague-Dawley; RT, room temperature; icv, intracerebroventricular; i.p., intraperitoneal injection; qRT-PCR, quantitative real-time polymerase chain reaction, ELISA, Enzyme-linked immune-sorbent assay; NC, negative control; H\&E, Hematoxylin and eosin; MWM, Morris water-maze; PBS, phosphatebuffered saline; RM, rat microglia; rcf, relative centrifugal force; NCBI, National Center for Biotechnology Information; GEO, Gene Expression Omnibus; WT, wildtype; MUT, mutant; PI, propidium iodide; SEM, standard error mean; mfe, minimum free energy.

\section{Author Contributions}

All authors made a significant contribution to the work reported, whether that is in the conception, study design, execution, acquisition of data, analysis and interpretation, or in all these areas; took part in drafting, revising or critically reviewing the article; gave final approval of the version to be published; have agreed on the journal to which the article has been submitted; and agree to be accountable for all aspects of the work.

\section{Funding}

This work is supported by the National Natural Science Foundation of China (81760208 and 81760261) and the Key Laboratory of Anesthesiology of Jiangxi Province (20164BCD40093).

\section{Disclosure}

The authors declare that no conflict of interest exists.

\section{References}

1. Azam S, Haque ME, Jakaria M, et al. G-Protein-coupled receptors in CNS: a potential therapeutic target for intervention in neurodegenerative disorders and associated cognitive deficits. Cells. 2020;9(2):506.

2. Erkkinen MG, Kim MO, Geschwind MD. Clinical neurology and epidemiology of the major neurodegenerative diseases. Cold Spring Harb Perspect Biol. 2018;10:4.

3. Kwon HS, Koh SH. Neuroinflammation in neurodegenerative disorders: the roles of microglia and astrocytes. Transl Neurodegener. 2020;9(1):42.

4. Calsolaro V, Edison P. Neuroinflammation in Alzheimer's disease: current evidence and future directions. Alzheimers Dement. 2016;12 (6):719-732.

5. Zuo Z. Postoperative cognitive effects in newborns: the role of inflammatory processes. Anesthesiology. 2013;118(3):481-483.

6. Wang J, Song Y, Chen Z, Leng SX. Connection between systemic inflammation and neuroinflammation underlies neuroprotective mechanism of several phytochemicals in neurodegenerative diseases. Oxid Med Cell Longev. 2018;2018:1972714. 
7. Zhao WX, Zhang JH, Cao JB, et al. Acetaminophen attenuates lipopolysaccharide-induced cognitive impairment through antioxidant activity. J Neuroinflammation. 2017;14(1):17.

8. Guan F, Zhou X, Li P, et al. MG53 attenuates lipopolysaccharideinduced neurotoxicity and neuroinflammation via inhibiting TLR4/ NF- $\kappa$ B pathway in vitro and in vivo. Prog Neuropsychopharmacol Biol Psychiatry. 2019;95:109684.

9. Xu M, Yan T, Gong G, et al. Purification, structural characterization, and cognitive improvement activity of a polysaccharides from Schisandra chinensis. Int J Biol Macromol. 2020;163:497-507.

10. Wu X, Lv YG, Du YF, et al. Inhibitory effect of INT-777 on lipopolysaccharide-induced cognitive impairment, neuroinflammation, apoptosis, and synaptic dysfunction in mice. Prog Neuropsychopharmacol Biol Psychiatry. 2019;88:360-374.

11. Garden GA, Möller T. Microglia biology in health and disease. J Neuroimmune Pharmacol. 2006;1(2):127-137.

12. de Araújo Boleti AP, de Oliveira Flores TM, Moreno SE, et al. Neuroinflammation: an overview of neurodegenerative and metabolic diseases and of biotechnological studies. Neurochem Int. 2020;136:104714.

13. Walsh JG, Muruve DA, Power C. Inflammasomes in the CNS. Nat Rev Neurosci. 2014;15(2):84-97.

14. Chen L, Dong R, Lu Y, et al. MicroRNA-146a protects against cognitive decline induced by surgical trauma by suppressing hippocampal neuroinflammation in mice. Brain Behav Immun. 2019;78:188-201.

15. Luo D, Liu F, Zhang J, et al. Functional crosstalk between Long non-coding RNAs and the NLRP3 inflammasome in the regulation of diseases. Mol Immunol. 2021.

16. Jiang W, Lv H, Wang H, et al. Activation of the NLRP3/caspase-1 inflammasome in human dental pulp tissue and human dental pulp fibroblasts. Cell Tissue Res. 2015;361(2):541-555.

17. Cattaneo A, Cattane N, Galluzzi S, et al. Association of brain amyloidosis with pro-inflammatory gut bacterial taxa and peripheral inflammation markers in cognitively impaired elderly. Neurobiol Aging. 2017;49:60-68.

18. Shen H, Guan Q, Zhang X, et al. New mechanism of neuroinflammation in Alzheimer's disease: the activation of NLRP3 inflammasome mediated by gut microbiota. Prog Neuropsychopharmacol Biol Psychiatry. 2020;100:109884.

19. Wang Z, Shiyu M, Lin C, et al. Critical role of NLRP3-caspase-1 pathway in age-dependent isoflurane-induced microglial inflammatory response and cognitive impairment. $J$ Neuroinflammation. 2018;15(1):109.

20. Fu Q, Li J, Qiu L, et al. Inhibiting NLRP3 inflammasome with MCC950 ameliorates perioperative neurocognitive disorders, suppressing neuroinflammation in the hippocampus in aged mice. Int Immunopharmacol. 2020;82:106317.

21. Shao A, Fei J, Feng S, Weng J. Chikusetsu saponin IVa alleviated sevoflurane-induced neuroinflammation and cognitive impairment by blocking NLRP3/caspase-1 pathway. Pharmacol Rep. 2020;72(4):833-845.

22. Wu YY, Kuo HC. Functional roles and networks of non-coding RNAs in the pathogenesis of neurodegenerative diseases. $J$ Biomed Sci. 2020;27(1):49.

23. He Z, Ruan X, Liu X, et al. FUS/circ_002136/miR-138-5p/SOX13 feedback loop regulates angiogenesis in Glioma. J Exp Clin Cancer Res. 2019;38(1):65.

24. Bai X, Shao J, Zhou S, et al. Inhibition of lung cancer growth and metastasis by DHA and its metabolite, RvD1, through miR-138-5p/ FOXC1 pathway. J Exp Clin Cancer Res. 2019;38(1):479.

25. Zhang D, Liu X, Zhang Q, Chen X. miR-138-5p inhibits the malignant progression of prostate cancer by targeting FOXC1. Cancer Cell Int. 2020;20:297.
26. Liu Y, Liu H, Li Y, et al. Circular RNA SAMD4A controls adipogenesis in obesity through the miR-138-5p/EZH2 axis. Theranostics. 2020;10(10):4705-4719.

27. Hu X, Ma R, Cao J, et al. CircSAMD4A aggravates H/R-induced cardiomyocyte apoptosis and inflammatory response by sponging miR-138-5p. J Cell Mol Med. 2020.

28. Zhou ZB, Du D, Huang GX, Chen A, Zhu L. Circular RNA Atp9b, a competing endogenous RNA, regulates the progression of osteoarthritis by targeting miR-138-5p. Gene. 2018;646:203-209.

29. Le Ribeuz H, Courboulin A, Ghigna MR, et al. In vivo miR-138-5p inhibition alleviates monocrotaline-induced pulmonary hypertension and normalizes pulmonary KCNK3 and SLC45A3 expression. Respir Res. 2020;21(1):186.

30. Büttnerennever $\mathrm{J}$. The rat brain in stereotaxic coordinates, 3rd edn. J ANAT. 1997; 191(Pt 2):315.

31. Peng J, Pang J, Huang L, et al. LRP1 activation attenuates white matter injury by modulating microglial polarization through Shc1/ PI3K/Akt pathway after subarachnoid hemorrhage in rats. Redox Biol. 2019;21:101121.

32. Tyagi E, Agrawal R, Nath C, Shukla R. Influence of LPS-induced neuroinflammation on acetylcholinesterase activity in rat brain. J Neuroimmunol. 2008;205(1-2):51-56.

33. Richard M. Developments of a water-maze procedure for studying spatial learning in the rat. J Neurosci Methods. 1984;11(1):47-60.

34. Xiao X, Yong L, Liu D, et al. Effects of in utero exposure to lanthanum on neurological behavior in rat offspring. Neurotoxicol Teratol. 2020;77:106854.

35. Sierksma AS, Prickaerts J, Chouliaras L, et al. Behavioral and neurobiological effects of prenatal stress exposure in male and female APPswe/PS1dE9 mice. Neurobiol Aging. 2013;34(1): 319-337.

36. Cai Q, Li Y, Pei G. Polysaccharides from Ganoderma lucidum attenuate microglia-mediated neuroinflammation and modulate microglial phagocytosis and behavioural response. $J$ Neuroinflammation. 2017; 14(1):63.

37. Livak KJ, Schmittgen TD. Analysis of relative gene expression data using real-time quantitative PCR and the 2(-Delta Delta $\mathrm{C}(\mathrm{T})$ ) Method. Methods. 2001;25(4):402-428.

38. Juźwik CA, Zhang SSD, et al. microRNA dysregulation in neurodegenerative diseases: a systematic review. Prog Neurobiol. 2019; 182:101664.

39. Zhao J, Bi W, Xiao S, et al. Neuroinflammation induced by lipopolysaccharide causes cognitive impairment in mice. Sci Rep. 2019;9 (1):5790.

40. Zhang XY, Xu ZP, Wang W, et al. Vitamin C alleviates LPS-induced cognitive impairment in mice by suppressing neuroinflammation and oxidative stress. Int Immunopharmacol. 2018;65:438-447.

41. Liu Y, Zhang Y, Zheng X, et al. Galantamine improves cognition, hippocampal inflammation, and synaptic plasticity impairments induced by lipopolysaccharide in mice. $J$ Neuroinflammation. 2018;15(1):112.

42. Glass CK, Saijo K, Winner B, Marchetto MC, Gage FH. Mechanisms underlying inflammation in neurodegeneration. Cell. 2010;140 (6):918-934.

43. Wei P, Yang F, Zheng Q, Tang W, Li J. The potential role of the NLRP3 inflammasome activation as a link between mitochondria ROS generation and neuroinflammation in postoperative cognitive dysfunction. Front Cell Neurosci. 2019;13:73.

44. Ge X, Guo M, Hu T, et al. Increased microglial exosomal miR-124$3 \mathrm{p}$ alleviates neurodegeneration and improves cognitive outcome after rmTBI. Mol Ther. 2020;28(2):503-522.

45. Cha DJ, Mengel D, Mustapic M, et al. miR-212 and miR-132 are downregulated in neurally derived plasma exosomes of Alzheimer's patients. Front Neurosci. 2019;13:1208. 
46. Ansari A, Maffioletti E, Milanesi E, et al. miR-146a and miR-181a are involved in the progression of mild cognitive impairment to Alzheimer's disease. Neurobiol Aging. 2019;82:102-109.

47. Zhang Y, Ai H, Fan X, et al. Knockdown of long non-coding RNA HOTAIR reverses cisplatin resistance of ovarian cancer cells through inhibiting miR-138-5p-regulated EZH2 and SIRT1. Biol Res. 2020;53 (1): 18 .

48. Li DW, Liu JZ, Li SC, et al. Fear conditioning downregulates miR-138 expression in the hippocampus to facilitate the formation of fear memory. Neuroreport. 2018;29(16):1418-1424.

49. Lau P, Bossers K, Janky R, et al. Alteration of the microRNA network during the progression of Alzheimer's disease. EMBO Mol Med. 2013;5(10):1613-1634.

50. Li D, Yang H, Ma J, et al. MicroRNA-30e regulates neuroinflammation in MPTP model of Parkinson's disease by targeting Nlrp3. Hum Cell. 2018;31(2):106-115.
51. Fan Z, Lu M, Qiao C, et al. MicroRNA-7 enhances subventricular zone neurogenesis by inhibiting NLRP3/Caspase-1 axis in adult neural stem cells. Mol Neurobiol. 2016;53(10):7057-7069.

52. Junn E, Lee KW, Jeong BS, et al. Repression of alpha-synuclein expression and toxicity by microRNA-7. Proc Natl Acad Sci USA. 2009;106(31):13052-13057.

53. Zhou Y, Lu M, Du R-H, et al. MicroRNA-7 targets Nod-like receptor protein 3 inflammasome to modulate neuroinflammation in the pathogenesis of Parkinson's disease. Mol Neurodegener. 2016;11:28.

54. Han C, Guo L, Yang Y, et al. Mechanism of microRNA-22 in regulating neuroinflammation in Alzheimer's disease. Brain Behav. 2020;10(6):e01627.

\section{Publish your work in this journal}

The Journal of Inflammation Research is an international, peerreviewed open-access journal that welcomes laboratory and clinical findings on the molecular basis, cell biology and pharmacology of inflammation including original research, reviews, symposium reports, hypothesis formation and commentaries on: acute/chronic inflammation; mediators of inflammation; cellular processes; molecular mechanisms; pharmacology and novel anti-inflammatory drugs; clinical conditions involving inflammation. The manuscript management system is completely online and includes a very quick and fair peerreview system. Visit http://www.dovepress.com/testimonials.php to read real quotes from published authors. 